\title{
Uncovering cancer vulnerabilities by machine learning prediction of synthetic lethality
}

Salvatore Benfatto ${ }^{1 \dagger}$, Özdemirhan Serçin ${ }^{1 \dagger}$, Francesca R. Dejure ${ }^{1 \dagger}$, Amir Abdollahi $^{2}$, Frank T. Zenke ${ }^{3}$ and Balca R. Mardin ${ }^{1 *}$

\begin{abstract}
Background: Synthetic lethality describes a genetic interaction between two perturbations, leading to cell death, whereas neither event alone has a significant effect on cell viability. This concept can be exploited to specifically target tumor cells. CRISPR viability screens have been widely employed to identify cancer vulnerabilities. However, an approach to systematically infer genetic interactions from viability screens is missing.

Methods: Here we describe PAn-canceR Inferred Synthetic lethalities (PARIS), a machine learning approach to identify cancer vulnerabilities. PARIS predicts synthetic lethal (SL) interactions by combining CRISPR viability screens with genomics and transcriptomics data across hundreds of cancer cell lines profiled within the Cancer Dependency Map.

Results: Using PARIS, we predicted 15 high confidence SL interactions within 549 DNA damage repair (DDR) genes. We show experimental validation of an SL interaction between the tumor suppressor CDKN2A, thymidine phosphorylase (TYMP) and the thymidylate synthase (TYMS), which may allow stratifying patients for treatment with TYMS inhibitors. Using genome-wide mapping of SL interactions for DDR genes, we unraveled a dependency between the aldehyde dehydrogenase ALDH2 and the BRCA-interacting protein BRIP1. Our results suggest BRIP1 as a potential therapeutic target in $\sim 30 \%$ of all tumors, which express low levels of ALDH2.
\end{abstract}

Conclusions: PARIS is an unbiased, scalable and easy to adapt platform to identify SL interactions that should aid in improving cancer therapy with increased availability of cancer genomics data.

\section{Introduction}

Synthetic lethality occurs when simultaneous perturbation in two or more genes leads to cell death, whereas individual inactivation of single genes is still compatible with cell survival [1]. This phenomenon, first described in Drosophila melanogaster [2,3], has been used as an approach in cancer therapy to exploit vulnerabilities of

\footnotetext{
*Correspondence: mardin@bio.mx

†Salvatore Benfatto, Özdemirhan Serçin and Francesca R. Dejure contributed equally to this work.

1 BioMed X Institute (GmbH), Im Neuenheimer Feld 583, 69120 Heidelberg, Germany

Full list of author information is available at the end of the article
}

cancer cells by identifying druggable targets that when ablated or inhibited would selectively impact the viability of aberrant cancer cells [4]. The first successful therapy based on this approach is the use of Poly-ADP-ribose polymerase 1 (PARP1) inhibitors in patients deficient in homologous recombination pathway [5]. This discovery promoted the search for additional synthetic lethal (SL) targets in cancer research.

Studies in human cancer cell lines have accumulated multiple layers of genetic information that can be used to study SL interactions. This includes CRISPR/Cas9-based KO screens, RNAi and drug screens together with gene expression, mutation and copy number variation data. For instance, data from the Achilles project (part of the original author(s) and the source, provide a link to the Creative Commons licence, and indicate if changes were made. The images or other third party material in this article are included in the article's Creative Commons licence, unless indicated otherwise in a credit line to the material. If material is not included in the article's Creative Commons licence and your intended use is not permitted by statutory regulation or exceeds the permitted use, you will need to obtain permission directly from the copyright holder. To view a copy of this licence, visit http://creativecommons.org/licenses/by/4.0/. The Creative Commons Public Domain Dedication waiver (http://creativeco mmons.org/publicdomain/zero/1.0/) applies to the data made available in this article, unless otherwise stated in a credit line to the data. 
DepMap consortium [6]), based on both CRISPR-Cas9 and shRNA screens, have been exploited to uncover new potential SL interactions and several computational approaches have been developed for this purpose [7-13]. In addition, prioritization of cancer therapeutic targets has been proposed [14] based on a priority score derived from experimental evidence, the significance of fitness deficiency, target gene expression, target mutational status and evidence for other fitness genes in the same pathway. Based on these efforts, recently the Werner syndrome helicase (WRN) was found as a promising SL target in microsatellite instable cancers [14-17]. Despite the depth of information acquired on individual cancer cell lines only a few potential SL interactions have been identified so far. Due to their genomic complexity, it has been challenging to disentangle the genetic dependencies of tumor cell lines. Several of the approaches used to infer SL interactions are based on the assumptions of gene co-inactivation, mutual exclusivity and/or co-expression and are generally tested on mutational or gene expression data with traditional statistics (e.g. Wilcoxon rank sum test) or restricted to certain tissues $[7,8,11]$.

Most of the current approaches for SL predictions are based on a massive number of multiple univariate statistical tests. This can lead to high number of false positives or after multiple-testing correction, to high number of false negatives. These models also do not address multicollinearity, a phenomenon in which the multiple predictors are highly correlated. For this reason, they may fail to predict potential feature associations by testing genetic interactions individually and they are not designed to find non-linear correlations. A major challenge is that the omics data are often composed of a relatively small number of samples and a high number of variables (often referred to as features or predictors). This type of data is also referred to as "big-p, little-n" $(p>>n)$, presents a problem of high dimensionality. A good strategy to tackle this problem is to reduce the number of dimensions through feature selection. By grouping similar features or filtering redundant ones, direct relationships between the response and the predictor can be readily identified. Feature selection methods can be grouped into (i) minimal-optimal methods that aim to find the minimal optimal subset of features to maximize the accuracy of the model, and (ii) all-relevant methods, that find all the relevant features to describe the outcome variable [18]. If the first ones often drop redundant (collinear) variables and suffer from inherent bias, the latter ones are able to capture all the important features to explain a phenomenon and have been successfully applied in bioinformatics, e.g. gene and single nucleotide polymorphism selection [19]. Random Forest (RF) algorithms are thus very suitable for $p>>\mathrm{n}$ datasets, they require minimal tuning, no transformation of variables and they are robust to noise [20].

In particular, the robustness of the RF methods has been previously tested with gene expression datasets, whereby they showed high reliability in terms of postselection classification accuracy and consistency.

Previous methods [7-13] provided very important steps towards the identification of targetable genetic interactions. For instance, Jerby-Arnon, L. et al. developed an approach (DAISY- data mining synthetic lethality identification pipeline) that led to the discovery of SL partners for the tumor suppressor VHL. Sinha, S. et al. presented MiSL (Mining Synthetic Lethals), an algorithm to identify mutation-specific SL partners in cancers and identified a SL interaction between the isocitrate dehydrogenase 1 (IDH1) mutation and the acetyl-CoA carboxylase 1 ( $A C A C A)$. Apaolaza, I. et al. employed the concept of genetic minimal cut sets and gene expression data to predict metabolic vulnerabilities in cancer and study the ribonucleotide reductase catalytic subunit M1 (RRM1) inhibition in myeloma cell lines. Lee, J. S. et al. developed an algorithm for identification of clinically relevant SL interactions (ISLE) using The Cancer Genome Atlas (TCGA) data. Despite these advances, an algorithm that can address the importance of each individual gene deficiency in explaining the dependencies observed in cancer cells in a tissue-independent manner is still missing. Here we present PAn-canceR Inferred Synthetic lethalities (PARIS), a machine learning approach that can predict potential vulnerabilities in cancer. The core of the workflow is a feature selection step, performed with $\mathrm{RF}$ algorithms that assign an importance score to each genomic feature based on the measured effect of knocking out a specific gene across hundreds of cancer cell lines. We initially established and tested PARIS to identify SL interactions among known DNA damage response (DDR) genes. We then expanded our search space and investigated the vulnerabilities of the DDR genes with the rest of the genome. We identified and confirmed two previously uncharacterized SL interactions. To allow straightforward exploration of the entire dataset, we generated an interactive web application. In summary, PARIS can be used as a platform to directly link certain genetic features to the viability data obtained after knocking out a specific gene and to uncover the meaningful relationships that represent potential cancer vulnerabilities.

\section{Results}

PARIS methodology

We developed PARIS as a computational approach to infer vulnerabilities in cancer cells. The core of the pipeline is based on the RF algorithms to assess importance of each independent variables (mutation or 


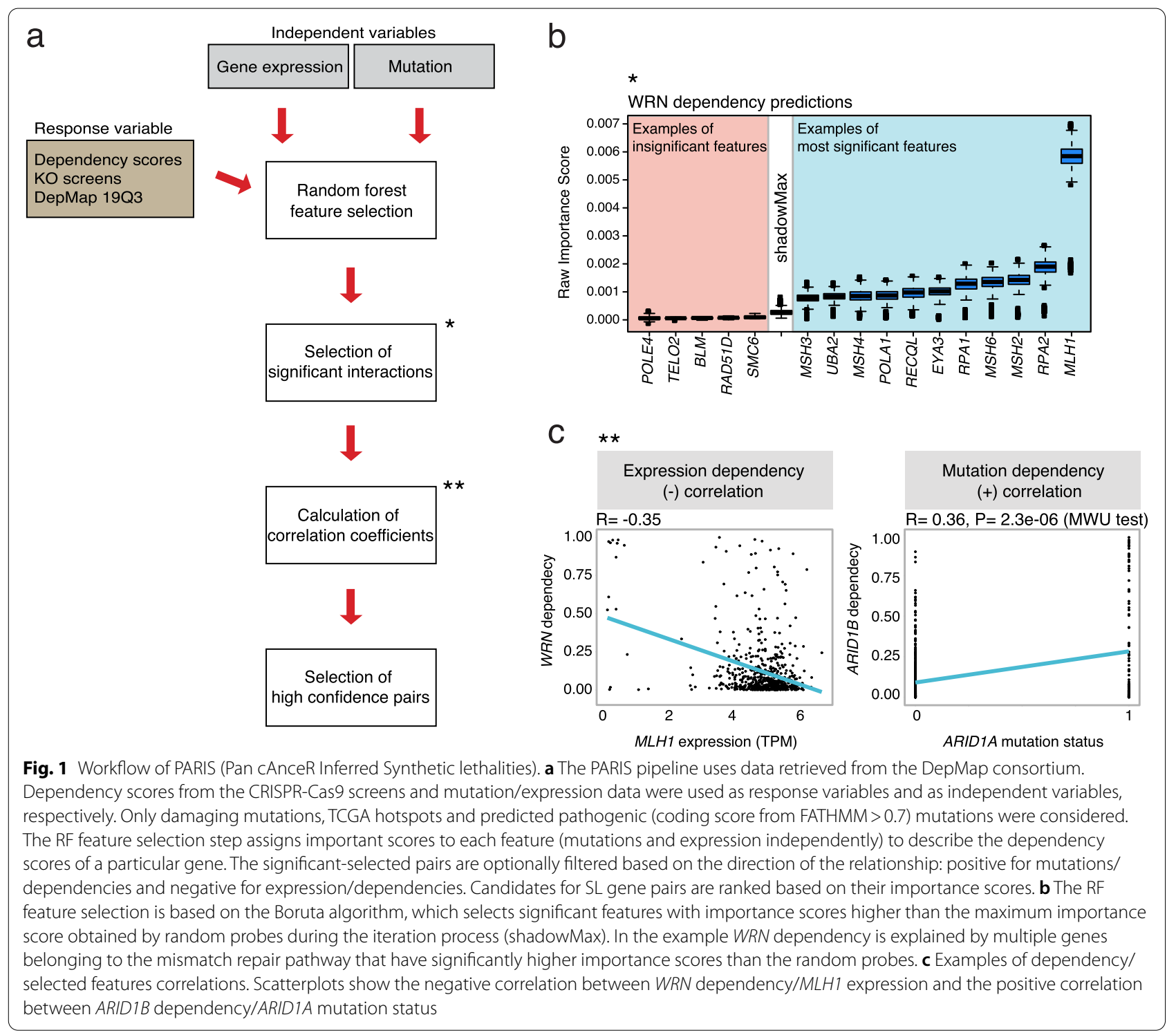

gene expression) with respect to the response variable (dependency scores). As the response variable, we retrieved gene dependency score data based on the CERES pipeline that can estimate the gene essentiality levels from CRISPR-Cas9 screen results correcting for the gRNA activity and the copy-number effect [21]. Additionally, we retrieved the mutation and expression data from the Cancer Cell Line Encyclopedia (CCLE), part of the DepMap consortium. In order to filter for relevant mutations, we only considered pathogenic mutations based on the FATHMM (Functional Analysis through Hidden Markov Models) predictions [22], hotspot mutations reported in TCGA or mutations that are already annotated as damaging. Using these data, we applied a feature selection step, based on a machine learning algorithm that aims to explain certain gene dependencies from the CRISPR-Cas9 screens by mutation or misregulation of genes across hundreds of cancer cell lines (Fig. 1a). For this purpose, we selected the Boruta algorithm [23] due to its robustness and adaptability to omics data [24]. This algorithm uses the RF importance scores and iteratively removes any features that are found to be significantly less relevant (i.e. show a lower importance score) than random probes (Fig. 1b).

To benchmark PARIS, we initially compared two importance measures to select significant features:

i) the mean decrease of impurity (Gini importance), obtained summing the total nodes impurity reduction where the variable appears, and ii) the mean decrease of accuracy (raw permutation importance), measuring 
accuracy reduction on out-of-bags samples when the values of the variable are randomly permuted.

\section{Quality assessment of the importance scores}

As a proof of concept, we initially focused on known DDR-related genes due to their relevance as potential targets in cancer treatment. We manually curated a list of 549 genes $[25,26]$ for which we used the dependency scores across 625 cancer cell lines as response variables, as well as the mutational and expression data as features/predictors. We then selected pathogenically mutated or misregulated genes that can explain the dependencies of each genetic target in the CRISPR-Cas9 screens using the Boruta algorithm. We extracted the selected pairs of genes along with the importance scores retrieved from the feature-selection step (Fig. 1b). For instance, we found that $M L H 1$ was one of the most significant features to explain WRN dependency, as previously described [14-17]. In order to identify interactions that could represent potential SL interactions or vulnerabilities in cancer cell lines, we focused on positive relationships in the case of dependency/mutation pairs and on negative relationships for dependency/expression pairs, using the Pearson correlation coefficient (PCC) score to retrieve the direction of the relationship. We used PCC due to its well suitability for data with high class imbalances such as the gene dependencies in cancer cells, whereby the dependent group is much smaller than the non-dependent group. For example, the WRN dependency scores negatively correlated with $M L H 1$ expression and ARID1B dependency score positively correlated with ARID1A mutation status, as expected from previous reports [27, 28], demonstrating the capability of PARIS to detect SL interactions (Fig. 1c). We selected all the significant importance scores and subsequently, we scaled the importance scores between 0 and 1 within each group, where the most significant interaction was scaled to 1 . We next investigated the concordance between the importance scores derived from different algorithms.
Considering only the interactions selected by both algorithms, the two scaled importance scores showed a strong correlation ( $\mathrm{R}=0.84, \mathrm{PCC})$ for predictions with mutations, however a moderate $(\mathrm{R}=0.58, \mathrm{PCC})$ correlation for the expression-based predictions (Fig. 2a). We reasoned that these differences may stem from the fact that the expression data allow many more splitting points during the tree construction process implemented by PARIS compared to the mutation-based data. Since the Gini metrics can be prone to assign higher scores on predictions based on the expression data, we hypothesized that it may introduce biases. For this reason, we applied an additional RF algorithm using a corrected impurity (corrected Gini) importance score [29] to improve the feature selection step (Fig. 2a). This metric is able to reduce biases in the measurement and is suggested to be as accurate as the raw permutation one with the advantage of reduced computational time (see Methods).

The scaled importance scores of the selected pairs of genes from the RF feature selection step showed longtailed distributions in all the groups (Supplementary Fig. 1a) indicating that most of the interactions were inferred with low importance scores and fewer relationships were assigned high scores. To select gene pairs with the highest confidence, we defined classes of importance scores based on the head/tail breaks clustering method, which is a clustering algorithm well suited for data with heavy-tailed distributions (see Methods, Fig. $2 b$ and Supplementary Fig. 1a).

For example, using the raw permutation method in the expression dataset, out of 5865 gene pairs that passed the $P$ value threshold $(P<0.01), 14$ gene pairs had scaled importance score of more than 0.25 . On the other hand, the corrected Gini method identified less gene pairs that passed the $P$ value cutoff, however more gene pairs were identified with higher importance scores (Fig. 2c).

Based on these analyses, we considered the last break point of the head/tail breaks clustering method as the threshold for identifying the gene pairs with the highest confidence. We then investigated the robustness of the

\footnotetext{
(See figure on next page.)

Fig. 2 Importance score quality assessment and potential synthetic lethal interactions among DDR genes. a Comparisons of importance score calculation methods on commonly selected DDR gene pairs. Gini-raw permutation scaled importance scores correlation using mutation features (green) and Gini/Gini corrected_raw permutation scaled importance scores correlation with expression features (blue). b Density distribution of the raw permutation scaled importance scores with superimposed breaks obtained by the Head/Tail breaks algorithm using raw permutation (yellow lines) and Gini corrected (dotted red lines) importance score methods. c Number of selected gene pairs above different scaled importance score cutoffs based on expression (blue) or mutation (green) features. $\mathbf{d}$ STRINGdb combined scores of interacting gene pairs selected with high confidence (scaled importance score $>0.4$ ) by the three approaches and with low confidence (scaled importance score $<0.4$ ) by all of the methods. e Percentage of interacting gene pairs over the selected ones in the four described groups. $\mathbf{f}$ Network of predicted SLs among DDR genes based on the raw permutation importance score. Each node represents a gene and each edge a relationship; the arrow starts from the mutated (green) or dysregulated gene (blue) and arrives to the gene showing an associated increased dependency score. The width is proportional to the absolute value of the Pearson correlation coefficient. The color of the node shows the median of the dependency score of the gene in a grey scale. Different arrow shapes show three levels of confidence scores based on the scaled importance scores
} 


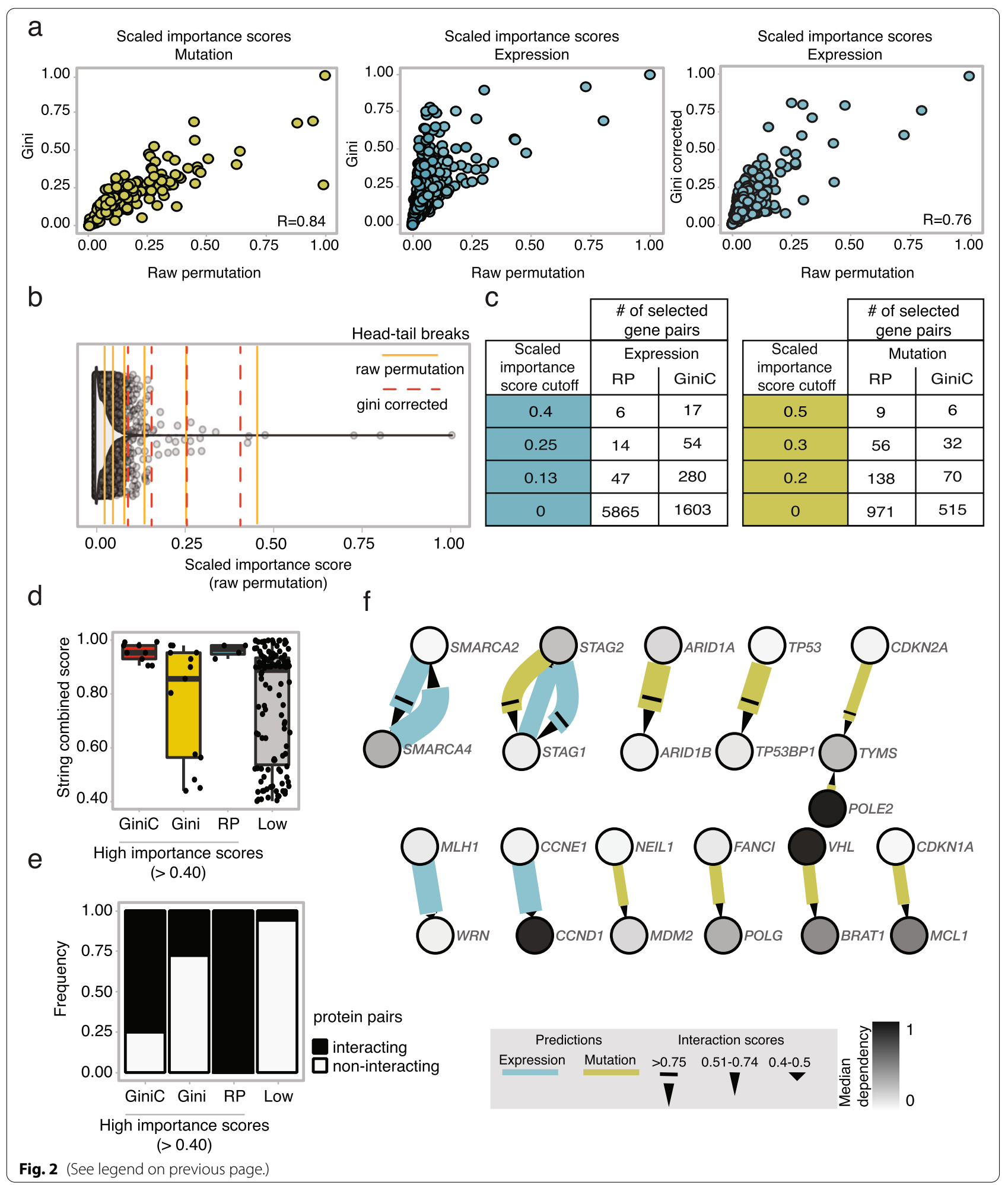


raw permutation, Gini and corrected Gini methods to identify interacting genes with high confidence (scaled importance score more than 0.4). For this we retrieved the interaction information from the STRING database [30], whenever available, as a proxy for the strength of the genetic interactions. In addition, we considered a fourth group including all the pairs selected with low confidence (scaled importance score less than 0.4 ) by all of the three approaches (Supplementary Fig. 1b). We first investigated the gene pairs identified from the expression-based data and compared the combined scores from the STRING database indicating the likelihood of protein-protein interactions. We observed higher combined scores in the groups belonging to the corrected Gini and the raw permutation scores than to the Gini and the "low confidence" groups (Fig. 2d). When we considered only the experiment-based interaction score, as the most stringent source of evidence, the raw permutation high confidence-selected pairs showed highest scores. However, in general, the percentage of interacting protein pairs was much higher in both the corrected Gini and raw permutation groups (Fig. 2e). As expected, we did not observe any differences among the three "high confidence" groups when we used the mutation data. Nonetheless, only the "low confidence" group showed lower values in terms of combined scores, experimentbased interaction score and percentage of interactions (Supplementary Fig. 2a, b). Based on these observations, we concluded that the raw permutation method is very robust in identifying gene pairs with high confidence and the corrected Gini method can drastically improve the confidence of selection, in particular when the expression data are used as independent variables. In addition, we demonstrated that the scaled importance scores can be an effective measure for the confidence of the predicted SL interactions by PARIS.

\section{Prediction of synthetic lethal interactions among DDR genes}

For intuitive data browsing and visualization we built an $\mathrm{R}$ shiny [31] app based on PARIS results. In this app, the selected gene pairs are represented as a directed graph, in which the arrows point to the dependent genes of the pairs starting from the deficient ones (mutated or dysregulated). Users can apply different filters, e.g. to the scaled importance scores and type of features to explore the interactions and export the results as data tables (see Methods for details and Additional file 1 for the complete table). In the context of the DDR genes, to select and investigate the pairs inferred with highest confidence, we used scaled importance score thresholds of 0.4 and 0.5 for expression and mutation-based predictions, respectively. Several of the gene pairs identified from this cohort are paralogs and function in protein complexes that are intrinsically related and more likely to show SL interactions (Supplementary Fig. 2c). As previously suggested, the buffering relationships between paralog pairs can explain a significant higher probability to display synthetic lethality, in agreement with our results in which the top high score pair group mostly consists of paralog pairs [32]. In addition, among others, notable examples of high confidence SL predictions based on mutation data were ARID1B-ARID1A, STAG1-STAG2 and TYMS-CDK2NA. When considering the expression data, we again observed a high confidence interaction between STAG1STAG2 in addition to a bidirectional interaction between SMARCA2 and SMARCA4 (Fig. 2f, Supplementary Fig. 3a, b). Interestingly, the combination of deficiencies of the genes ARID1B-ARID1A and SMARCA2-SMARC4/ SMARCA4-SMARCA2, all belonging to the SWI/SNF chromatin remodeling complex, has already been demonstrated to show SL interactions [27, 28, 33-35]. Additionally, the SL interaction between STAG1-STAG2 has previously been described [36].

\section{PARIS predicts TYMS dependency of cancer cell lines}

Having established that PARIS can identify high confidence SL interactions, we next focused on the previously uncharacterized TYMS-CDKN2A vulnerability identified in our analysis. PARIS predicted cells with $C D K N 2 A$ damaging mutations to show sensitivity to TYMS knockout (Fig. 2f, and Fig. 3a). The cyclin dependent kinase inhibitor 2A $(C D K N 2 A)$ is a ubiquitously expressed tumor suppressor gene. It encodes two proteins, p14 ${ }^{\text {Arf }}$ and $\mathrm{p} 16^{\text {INKA }}$, also referred to as $\mathrm{p} 14$ and $\mathrm{p} 16$, respectively. Both proteins act as tumor suppressors and are involved in cell cycle regulation. In the case of inactivating germline mutations, CDKN2A is one of the DDR-related genes associated with inherited/familial predisposition for melanoma, glioblastoma multiforme and pancreatic cancer. In addition, $C D K N 2 A$ is also frequently somatically mutated in various cancers [25]. The roles of CDKN2A in cell cycle pathways make it crucial for maintaining genomic stability and as a result, aberrations of it leads to defects in cell cycle regulation and senescence, contributing to tumorigenesis and poor disease prognosis [37]. Thymidylate Synthase (TYMS) is the enzyme that converts deoxyuridine monophosphate (dUMP) into deoxythymidine monophosphate (dTMP). This step is followed by the phosphorylation of dTMP to dTTP, which is in turn incorporated into DNA during DNA synthesis. TYMS plays an important role in replenishing the nucleotide pool for replication. Due to its pivotal role in the de novo synthesis of pyrimidines, TYMS is an established drug target for cancer treatment [38]. For instance, a new generation of antifolates targeting TYMS 
such as Pemetrexed (PMX) or Raltitrexed (RTX) are used for the treatment of squamous cell carcinomas of the lung and mesotheliomas among many other tumors [39, 40]. In addition, overexpression of TYMS is associated with resistance to PMX. However, how TYMS levels are regulated in cancer is not yet well understood [41, 42].

To confirm the PARIS predictions and test the sensitivity of cancer cells to TYMS inhibition, we treated a panel of cell lines with different $C D K N 2 A$ genetic backgrounds with increasing doses of PMX (ranging from $10 \mathrm{nM}$ to $15 \mu \mathrm{M})$ and measured cell viability as a readout for synthetic lethality. Even though the PARIS predictions were based on pathogenic mutations of $C D K N 2 A$, we observed pronounced PMX sensitivity not only in cell lines that carry a nonsense mutation in the CDKN2A gene (e.g. CAL27) but also in cell lines that have a homozygous $C D K N 2 A$ deletion (e.g. H292), whereas this effect was not observed in any CDKN2A-proficient cell lines (Fig. 3b,c). Importantly, the IC50 values derived from these assays varied significantly between the CDKN2A proficient and deficient cells with a median IC50 of $51.77 \mu \mathrm{M}$ for CDKN2A-proficient cells and $0.59 \mu \mathrm{M}$ for CDKN2Adeficient cells $(P=0.004$, Mann-Whitney $\mathrm{U}$ test). To better understand the response of CDKN2A mutant or deleted cancer cell lines to PMX we next performed immunoblots of the proteins involved in the thymidine nucleotide metabolism pathway, including TYMS, Thymidine phosphorylase (TYMP), thymidine kinase 1 (TK1), glycinamide ribonucleotide formyltransferase (GART) and dihydrofolate reductase (DHFR) as well as cleaved PARP1, and p-CHK1 as markers of apoptosis and DNA damage checkpoint activation, respectively. Upon PMX treatment, irrespective of their CDKN2A status, we observed that cells upregulated TYMS, TK1 and DHFR protein levels at least by twofold (Fig. 3d and Additional file 2). TYMS, TK1 and DHFR levels are known to be regulated during cell cycle [43-45] and the increase in their protein levels is possibly due to accumulation of cells in $S$ phase upon PMX, since we observed increased nuclear CyclinA levels following PMX treatment (Supplementary Fig. 4a). Interestingly, we observed higher baseline GART levels in CDKN2A- proficient cells (Fig. 3d, Additional file 2). Consistent with the viability results, $C D K N 2 A$ deficient cells that are most affected by PMX treatment such as CAL27, CAL62 and NCI-H292, strongly induced apoptosis upon PMX treatment (Fig. 3d). Importantly, sensitivity to either low $(50 \mathrm{nM})$ or high doses $(5 \mu \mathrm{M})$ of PMX in these cells was rescued by thymidine supplementation, as previously suggested [39] (Fig. 3e, Supplementary Fig. 4b). Thymidine can be converted into dTMP by both the cytosolic and mitochondrial enzymes thymidine kinases (TK1 and TK2, respectively) [46], thus strongly

\footnotetext{
(See figure on next page.)

Fig. 3 Synthetic Lethal interaction between CDKN2A and TYMP with TYMS.a Violin plot showing TYMS dependency (0 lowest, 1 highest) with respect to mutation status of CDKN2A in DepMap data. Each point represents corresponding cell line and dependency value. $P$-value was calculated using Mann-Whitney U test. b A panel of cancer cell lines carrying wildtype CDKN2A: MDA-MB-157, HCC1937, missense mutant: DU-145, NCI-H1703, nonsense mutant: CAL27, deleted for CDKN2A locus: CAL62, HOP62, NCI-H292, KYSE-140, KYSE-70, KYSE-450, splice site mutant: M14, were tested with increasing concentrations of $\operatorname{PMX}(0,0.001,0.01,0.05,0.1,0.25,0.5,1,5$ and $15 \mu \mathrm{M})$. Cell viability was measured with CellTiter Glo after incubation of the cells with PMX for $96 \mathrm{~h}$. Drug response curves were generated and IC50 values shown in brackets ( $\mu \mathrm{M})$ next to each cell line were calculated from at least 3 biological and 9 technical repeats. $\mathbf{c}$ Heatmap showing CDKN2A mutation status (red box $=$ nonsense mutation, green boxes = missense mutation, white boxes $=$ WT); CDKN2A, TYMP, TYMS, GART, DHFR expression status for cell lines used in this study. Color scale corresponds to $(\log 2(T P M)+1)$ values based on RNA-Seq. $\mathbf{d}$ Cancer cell lines were treated with PBS or $5 \mu M$ PMX for 48 h. Western blot was performed for the proteins involved in Thymidine nucleotide metabolism (DHFR, GART, TYMS, TK1 and TYMP), DNA damage checkpoint marker (phospho-CHK1 (S345)) and apoptosis marker (cleaved-PARP1). VINCULIN served as a loading control. Cell lines labeled with red color are CDKN2A-deficient and show sensitivity to PMX in (b). Quantification of these blots are available in Additional file 2. e PMX-sensitive cancer cell lines were supplemented with PBS or $50 \mu \mathrm{M}$ of thymidine to the media during PMX ( $50 \mathrm{nM}$ or $5 \mu \mathrm{M}$ ) treatment. Cell viability was measured using live-cell protease (CellTiter Fluor) and \% viability was calculated compared to the control treatment. Boxplots were generated from data from at least 3 biological and technical repeats. In the boxplots, centerlines mark the medians, box limits indicate the $25^{\text {th }}$ and $75^{\text {th }}$ percentiles, and whiskers extend to $5^{\text {th }}$ and $95^{\text {th }}$ percentiles. P-values were calculated using Mann-Whitney $U$ test. f CAL27 and CAL62 cells were transfected with gRNA targeting TYMP. Five days post transfection, control or TYMP KO cells were treated with increasing doses of PMX $(0,0,25,0.5,1,5$ and $15 \mu \mathrm{M})$ for 96 h. Drug response curves were generated using data from 8 and 2 biological replicates, respectively. (Right) Western blot analysis of the indicated proteins 5 days post gRNA transfection. g MDA-MB-157 cells were transfected with gRNA targeting TYMP, CDKN2A or both genes. Five days post transfection, control or KO cells were treated as described in (f). Drug response curves were generated from at least 3 biological replicates. (Right) Western blot analysis of the indicated proteins 5 days post gRNA transfection. $\mathbf{h}$ RPE1 TP53-I-;CMYC, RPE1 TP53-I-;CMYC;CDKN2A-/-, RPE1 TP53-l-; CMYC;TYMP, RPE1 ${ }^{\text {TPS3-I-; CMYC; TYMP;CDKN2A-/- }}$, cells were transfected with two different gRNA against TYMS and viability were measured 7 days using CellTiter Glo. Values were normalized to scrambled gRNA transfection and were plotted from at least 9 biological replicates. In the boxplots, centerlines mark the medians, box limits indicate the $25^{\text {th }}$ and $75^{\text {th }}$ percentiles, and whiskers extend to $5^{\text {th }}$ and $95^{\text {th }}$ percentiles. $P$-values were calculated using a MannWhitney $U$ test. (Right) Western blot analysis of the generated RPE-1 cell lines. i Prediction of TYMS dependency by different genetic backgrounds. DepMap cancer cell lines grouped by their CDKN2A mutation and TYMP expression status. In each group the ratios of the percentage of TYMS dependent/TYMS independent cell lines were calculated and plotted. $\mathbf{j}$ TYMS dependency distribution is shown as boxplots. Cell lines are grouped by their CDKN2A and TYMP expression status. CDKN2A deficiency/proficiency is defined by the presence of a mutation or copy number loss and TYMP status is defined by tissue as high and low expressed using the median as a cut-off
} 
a

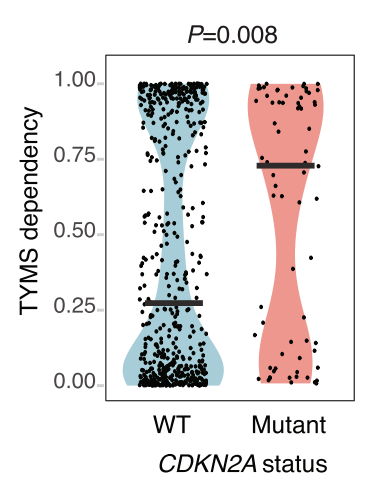

b

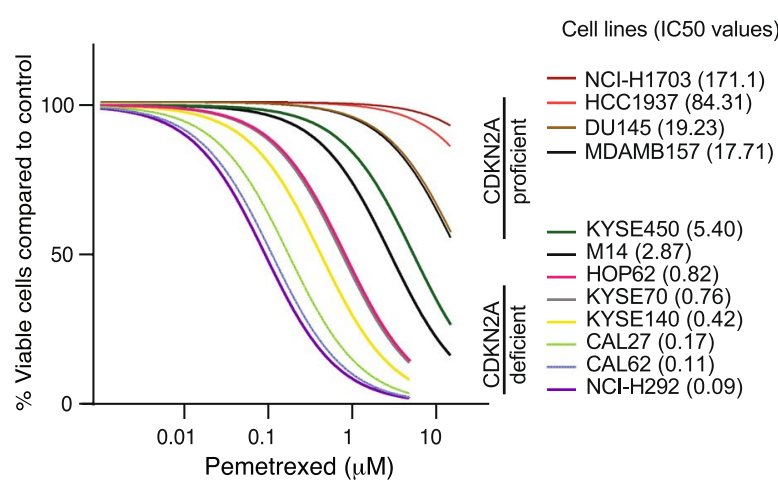

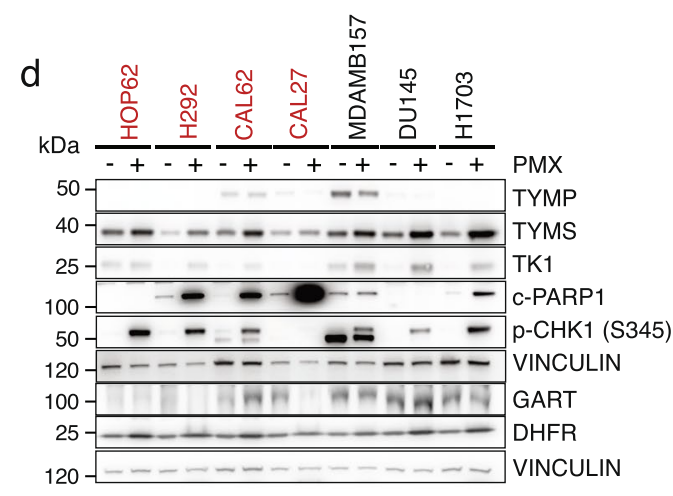

C

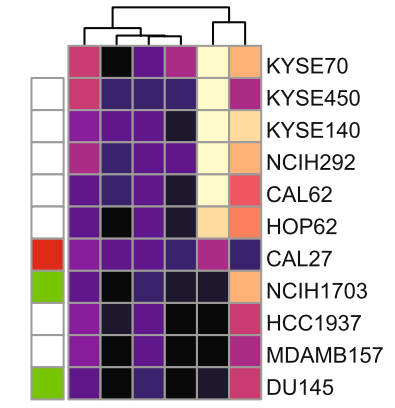

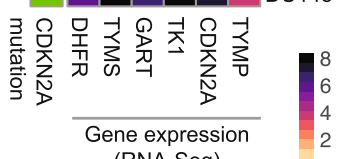
(RNA-Seq)

e

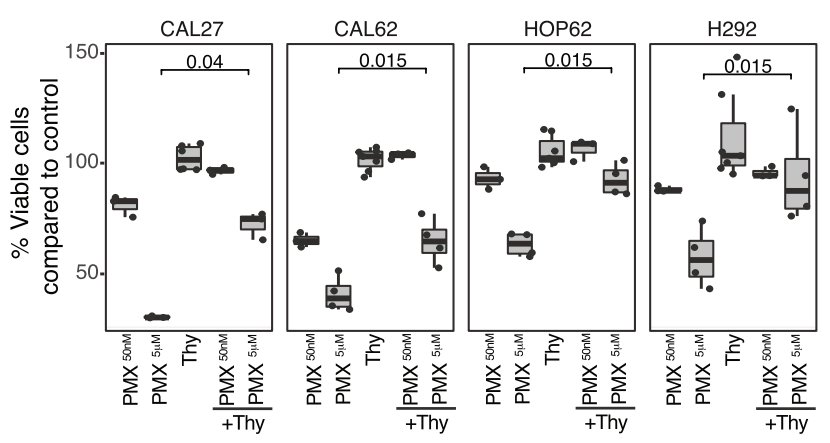

f

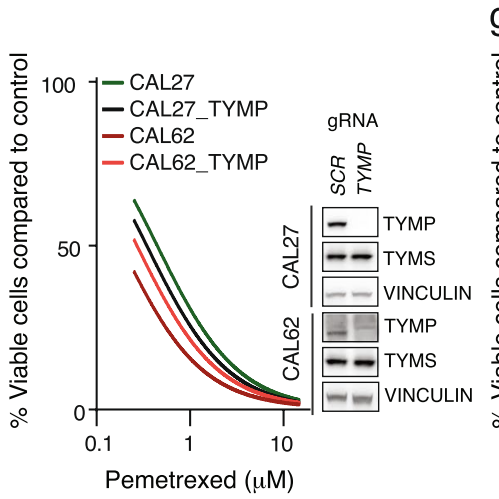

i

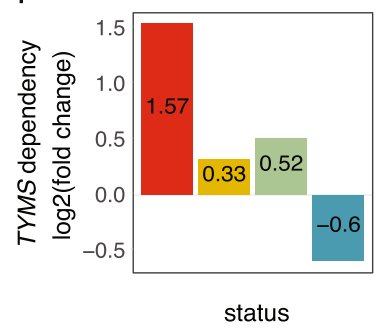

status

- CDKN2A deficienthigh TYMP

CDKN2A proficienthigh TYMP

- CDKN2A deficientlow TYMP

- CDKN2A proficientlow TYMP
MDA-MB-157

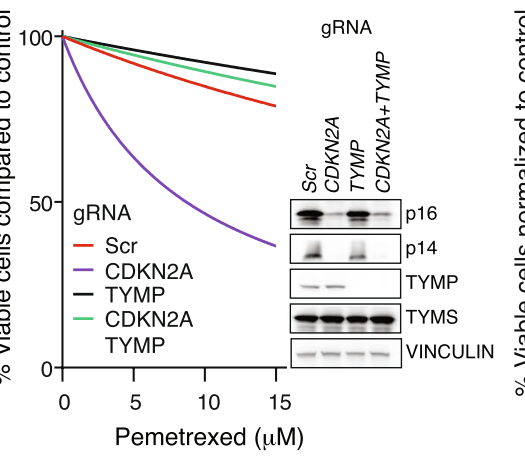

j
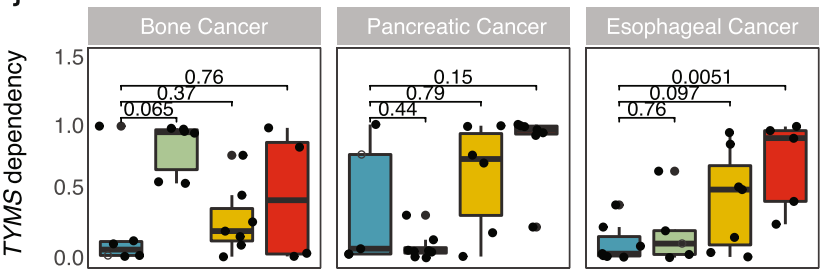

19 CDKN2A proficient-low TYMP CDKN2A proficient-high TYMP 审 CDKN2A deficient-low TYMP $C D K N 2 A$ deficient-high TYMP

Fig. 3 (See legend on previous page.) 
suggesting that the cell death occurs due to the depletion of dTMP through the predicted SL interaction.

Notably, TYMP overexpression was observed in some of the cell lines that show increased sensitivity to PMX, such as CAL27 and CAL62, which carry either a nonsense mutation in the $C D K N 2 A$ gene or a deletion of the CDKN2A locus, respectively (Fig. 3c). It has been reported that cells overexpressing TYMP are more sensitive to TYMS depletion [47]. TYMP functions within the pyrimidine salvage pathway by converting thymidine into thymine, thus it plays a key role in regulating thymidine levels and dTMP production [48]. Since PARIS also predicted that TYMP overexpression is linked to TYMS dependency (Supplementary Fig. 4c-f), we tested the direct involvement of TYMP overexpression in PMX sensitivity in the CAL27 and CAL62 cell lines that are $C D K N 2 A$-deficient. Transfection of gRNAs targeting TYMP in CAL27 or CAL62 cells stably expressing Cas9 did not lead to any changes in PMX sensitivity as compared to the control (scrambled) gRNA transfected cells, suggesting that TYMP expression alone is not sufficient to control TYMS dependency (Fig. 3f). To delineate the involvement of TYMP and p14/p16 in regulating PMX sensitivity, we used the MDA-MB-157 cell line, in which we observed sufficiently high levels of p14/p16 and TYMP and transfected gRNAs targeting TYMP and $C D K N 2 A$. While depletion of TYMP showed no additional sensitization to PMX, depletion of CDKN2A dramatically increased the sensitivity of these cells to PMX. This could be due to misincorporation of deoxyuridine during DNA synthesis and apoptosis upon PMX treatment or replication stress due to dTTP depletion as indicated by increased cleavage of PARP-1 and accumulation of cells in S phase (Fig. 3g, Supplementary Fig. 4 g, h). Strikingly, knockout (KO) of TYMP in CDKN2A-deleted MDA-MB-157 cells rescued their sensitivity to PMX and decreased apoptosis, suggesting a genetic interaction between TYMP and CDKN2A (Fig. 3g, Supplementary Fig. 4 g).

While PMX's known primary target is TYMS, at higher concentrations it is also known to inhibit additional enzymes such as DHFR and to a lesser extent
GART. To study the relative contributions of TYMP and $C D K N 2 A$ without PMX in a stable genetic background, we established a model cell line that resembles tumorigenic growth based on retinal pigment epithelial-1 (RPE1) cells by stable overexpression of $c-m y c$ and generated isogenic cell lines with CDKN2A deletion and/or TYMP overexpression. In these cell lines we then tested the effects of TYMS knockout on cell viability. In CDKN2A KO cells gRNAs targeting TYMS led to a mild but significant decrease in cell viability. The negative effect on cell viability was also observed by TYMP overexpression and was significantly exacerbated by combined overexpression of TYMP and CDKN2A deficiency (Fig. 3h, Supplementary Fig. 4i). Collectively, these results point to an effect of CDKN2A depletion on PMX sensitivity together with $T Y M P$. Indeed, categorizing DepMap data into 4 groups based on the CDKN2A status and TYMP gene expression levels, combination of CDKN2A deficiency and high TYMP expression best explains the TYMS sensitivity (Fig. 3i). However, it is possible that in different cell types, relative contributions of TYMP and CDKN2A to PMX sensitivity vary. Consistent with this idea, upon grouping the DepMap cancer cell lines according to tissue origin, we noted that CDKN2A status and TYMP expression have varying effects on controlling TYMS dependency in different tumor types. For instance, in bone cancer cell lines, deficiency of CDKN2A alone can explain the dependency to TYMS. On the other hand, dependency to TYMS in CDKN2A-mutant pancreatic cancer lines correlates with TYMP expression levels, while in esophageal cancer cell lines both TYMP expression levels and $C D K N 2 A$ status together can better explain TYMS sensitivity (Fig. 3j, Supplementary Fig. 5 and 6). A notable exception was observed in lung tumor cell lines in which neither TYMP expression nor $C D K N 2 A$ status affected the dependence of the cells to TYMS depletion (Supplementary Fig. 5 and 6). In summary, we propose that cells with $C D K N 2 A$ deficiency and TYMP overexpression together contribute to TYMS sensitivity and that these dependencies can be cell-type specific.

\footnotetext{
(See figure on next page.)

Fig. 4 Vulnerabilities of DDR related genes. a Network of predicted SLs between DDR genes and the genome based on the raw permutation importance score. Each node represents a gene and each edge a relationship; the arrow starts from the mutated (green) or dysregulated gene (blue) and arrives to the dependent gene. The width is proportional to the absolute value of the Pearson correlation coefficient. The color of the node shows the median of the dependency score of the gene in a grey scale. Different arrow shapes show three levels of confidence based on the scaled importance score. $\mathbf{b}$ Scaled raw permutation importance score distributions of selected gene pairs divided into paralogs or not in the two cohorts (expression and mutation). c Bar plots showing examples of high-confidence predicted SL gene pairs using expression features. The ranked bars show the dependency scores (mean centered) of one gene across the cancer cell lines and the color gradient shows the expression level of the second gene. $\mathbf{d}$ Scatterplots showing the gene expression levels (based on RNA-Seq) of BRIP1 and ALDH2 in matched tumor (TCGA) and normal (GTEX) breast, lung and brain tissues. e Top altered pathways in the enrichment analysis of differentially expressed genes in TCGA cc samples expressing high or low ALDH2. f Heatmap showing expression levels (mean centered) of the main Fanconi anemia genes in TCGA breast cancer samples expressing high or low ALDH2. g Scatterplots indicating the correlation between the expression levels of ALDH2 and BRIP1 gene effect together with the promoter methylation levels of $A L D H 2$ in breast cancer cell lines
} 
a
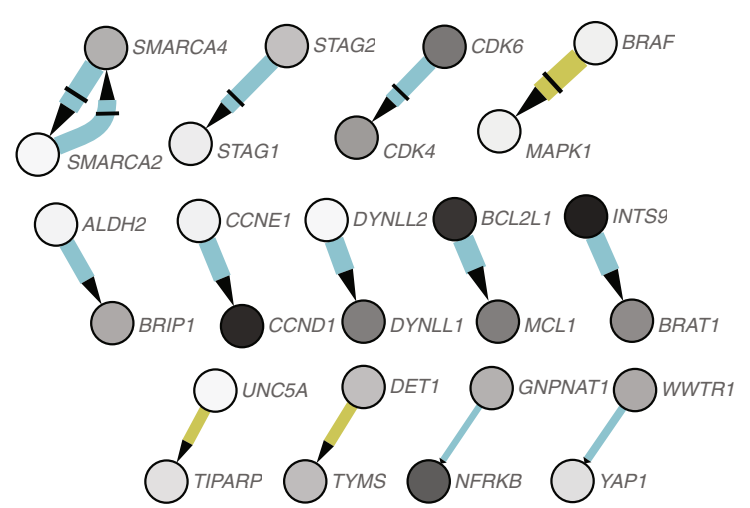

b
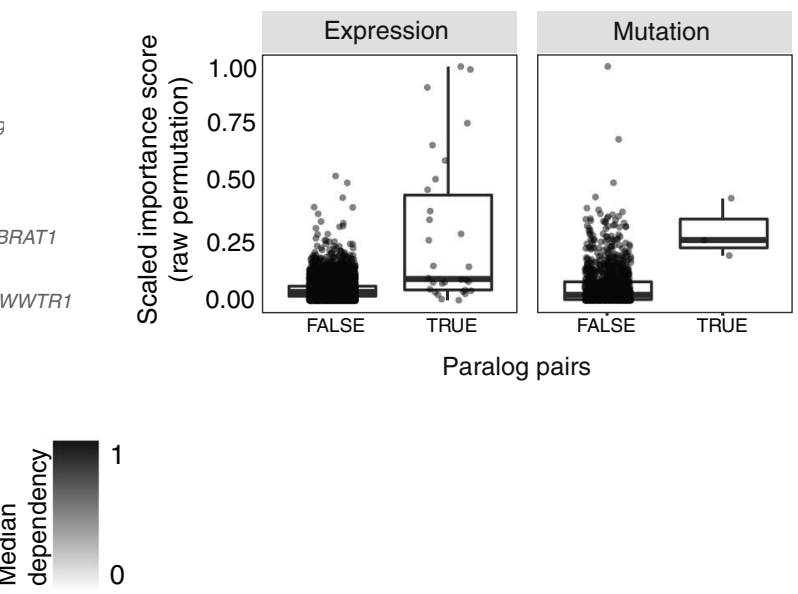

C
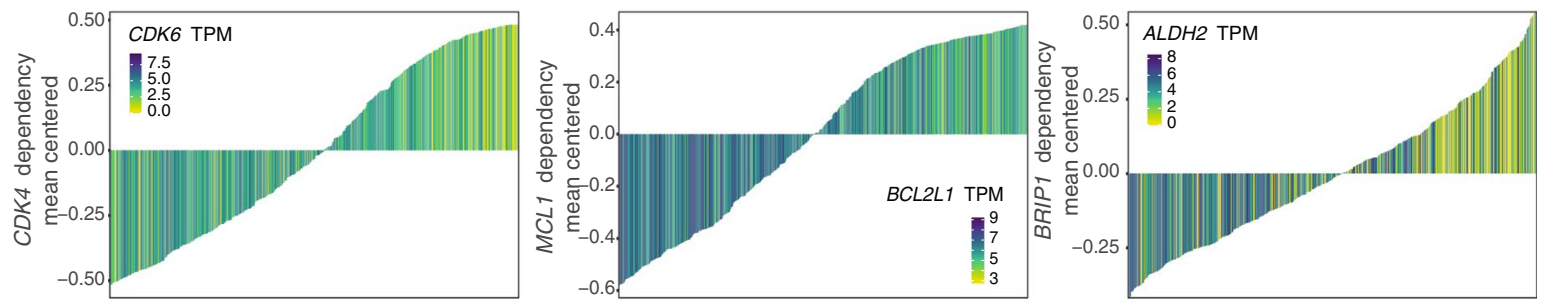

d
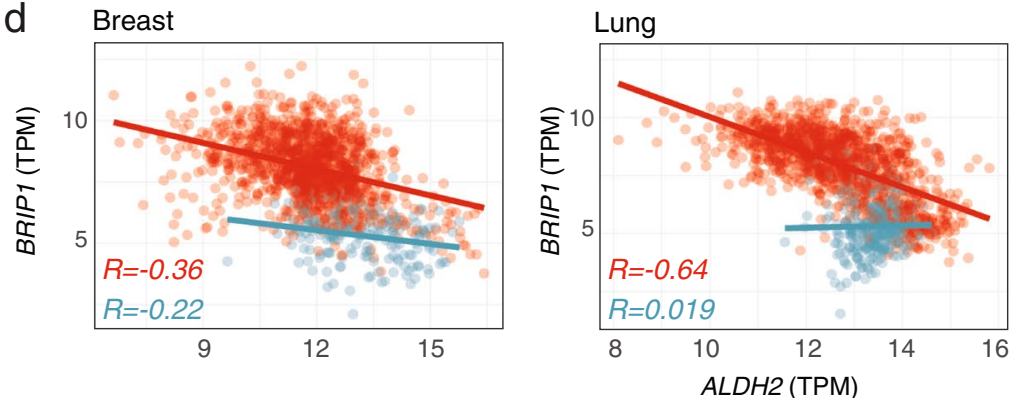

e

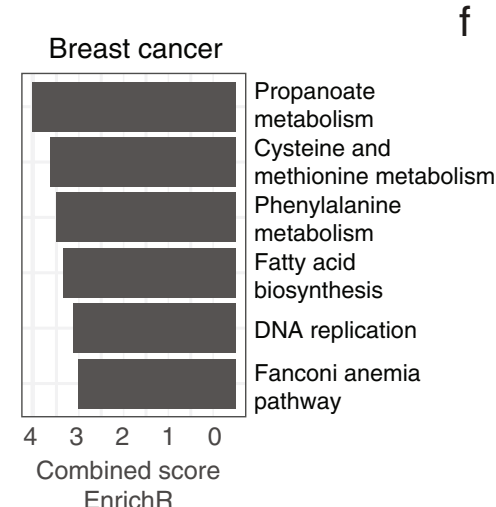

ALDH2 (low) ALDH2 (high)

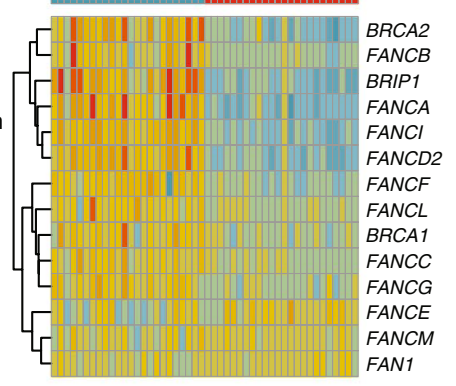

Expression

mean centered

$3210,2,3$

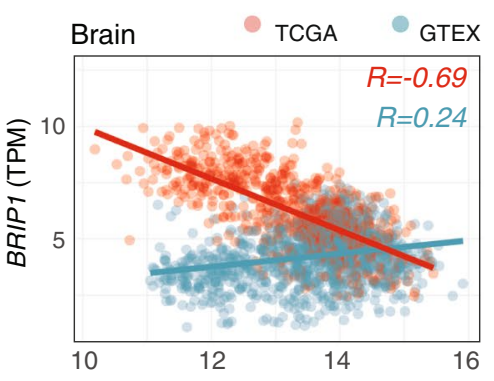

9 Breast Cancer Cell Lines

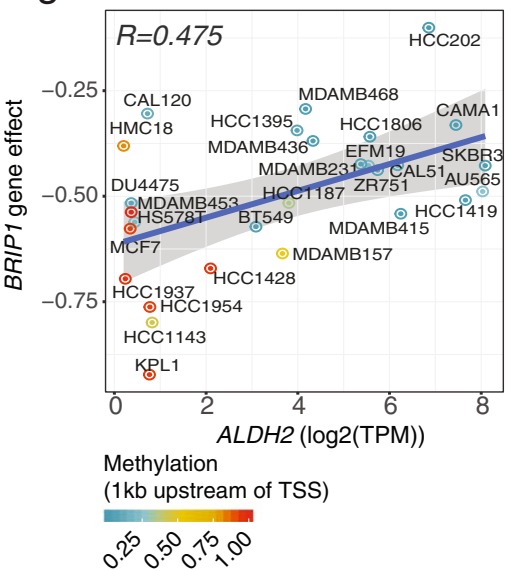

Fig. 4 (See legend on previous page.) 


\section{Prediction of vulnerabilities of DDR genes across the genome by PARIS}

To demonstrate the ability of PARIS to select SL interactions in larger cohorts and to reveal interesting potential vulnerabilities among DDR and other genes, we extended the features of the dataset (both for mutation and expression) to all the available genes $(\sim 18,000)$ (Additional file 3$)$. In this case, the Boruta algorithm with the raw permutation score was used to select the high-confidence pairs due to its reliability, as described above. Overall, the importance scores derived from the two cohorts' predictions were consistent (Supplementary Fig. 7). For instance, ARID1B-ARID1A, SMARCA2-SMARCA4 and STAG1-STAG2 were also found as high-confidence predicted SLs in this larger cohort (Fig. 4a). Moreover, we identified well-known vulnerabilities in cancer, such as MAPK1 dependency in BRAF mutated cells [49]. In agreement with the analysis performed within the DDR cohort, several of the predicted SL interactions are paralog genes (Fig. 4b), for instance CDK4-CDK6 [50]. Besides these paralog pairs, PARIS also predicted with high-confidence recently identified SL interactions such as the one between the anti-apoptotic genes MCL1 and BCL2L1 [51-54] (Fig. 4c). In general, and consistent with earlier results, we predicted more SL interactions based on the expression data, suggesting that expression is a better predictor to explain the dependency scores from the CRISPR-Cas9 screens [55].

Among these high-confidence SL pairs, we identified a potential new vulnerability between $A L D H 2$ and BRIP1 (Fig. 4c). The aldehyde dehydrogenase $2(A L D H 2)$ gene encodes a mitochondrial enzyme mainly involved in the detoxification of acetaldehyde [56]. BRCA1 interacting protein C-terminal helicase 1 (BRIP1) encodes a DNA helicase, also known as Fanconi Anemia group J protein (FANCJ), which plays a role within the Fanconi Anemia (FA) DNA repair pathway and has overall a broader function in maintaining genome stability by resolving DNA secondary structures [57]. The FA pathway is responsible for repairing interstrand crosslink (ICL) DNA damage, a type of lesion represented by a covalent bond between two complementary DNA strands. Such crosslinks cause genomic instability by interfering with the function of the replication machinery [58]. Acetaldehyde (ACE) is a highly reactive molecule produced from either exogenous sources, such as alcohol, cigarette smoke, environmental pollutants, or endogenous ones, such as intracellular metabolic reaction [59] and it represents a potential source of ICL formation $[60,61]$.

It has been shown that in hematopoietic stem cells, ALDH2 and the FA pathway provide two layers of protection against ACE-induced DNA damage [62-65], demonstrating an interesting link between the two pathways in the stem cell pool. However, the interplay between ALDH2 and BRIP1 or other components of the FA pathway in human tumors is less investigated. Based on the PARIS prediction, cancer cell lines that express low levels of $A L D H 2$ become dependent on BRIP1, possibly to balance a harmful increase in genomic instability. To understand how $A L D H 2$ and BRIP1 levels are regulated in human tumors, we looked at cancer gene expression data obtained from the TCGA and compared them to those found in the normal tissue controls derived from the Genotype-Tissue Expression (GTEx) database. We found $A L D H 2$ to be downregulated in several tumor types. Moreover, we observed, particularly in breast, brain and lung cancer cohorts, a strong negative correlation between $A L D H 2$ and BRIP1 (Fig. 4d). These data suggested a potential upregulation of BRIP1 in ALDH2low expressing tumor samples. Indeed, when we divided tumor samples based on their $A L D H 2$ expression levels and performed differential gene expression analysis, the FA pathway was one of the highly upregulated pathways among these two groups (Fig. 4e, Supplementary Fig. 8a, b), and the FA pathway components BRIP1, FANCD2 and $F A N C I$ were consistently upregulated across different tumor types (Fig. 4f, Supplementary Fig. 8c, d) and independently of tumor stage (Supplementary Fig. 8e). These results suggest that the $A L D H 2-B R I P 1$ vulnerability is important for a variety of tissue-specific cancers.

To further validate this prediction, we selected a panel of breast cancer cell lines to test the vulnerability of low $A L D H 2$ expressing cells. Interestingly, several of the breast cancer cell lines showed promoter hypermethylation of $A L D H 2$, suggesting an epigenetic control on the expression levels of $A L D H 2$ (Fig. 4g). To confirm these data, we assessed by RT-qPCR the expression of both $B R I P 1$ and $A L D H 2$ in 9 breast cancer cell lines representing different breast tumor subgroups [66] (Supplementary Fig. 9a). In 7 out of 9 cell lines, we measured low to undetectable levels of $A L D H 2$ mRNA, along with increased BRIP1 expression, recapitulating the inverse pattern of expression highlighted by PARIS. Consistent with the gene expression analysis, we observed high ALDH2 protein levels in SK-BR-3 and MDA-MB-468 and almost undetectable levels in MCF-7, HCC1954, and HCC1937 (Fig. 5a). Conversely, cell lines with low ALDH2 protein levels show increased BRIP1 protein as compared to SK-BR-3 and MDA-MB-468 (Fig. 5a).

To investigate if the predicted dependency on BRIP 1 in the absence of $A L D H 2$ expression represents a potential tumor vulnerability, we divided breast cancer cell lines into two groups: i) defined as the "control group", comprises SK-BR-3 and MDA-MB-468, which express high levels of $A L D H 2$; ii) defined as the "dependency group", 
comprises MCF-7, HCC1954, and HCC1937, which show low levels of $A L D H 2$ (and an inverse pattern of BRIP1 expression). Next, we tested the capability of our model cell lines to form colonies upon transfection of two independent gRNAs targeting BRIP1, both of which showed consistent downregulation of BRIP1 protein levels. We did not apply exogenous ACE as additional stress since the predicted data were retrieved from CRISPR-Cas9 screens performed in the absence of drug treatments and endogenously-produced ACE can provide a sufficient stimulus to trigger DNA damage [63, 65]. Consistent with our prediction, we observed a significant reduction ( $p \leq 0.01$, Mann-Whitney U test) in the number of colonies upon BRIP1 KO in the three cell lines belonging to the dependency group, while mild or no effects were observed in the control group cell lines (Fig. 5b, c, Supplementary Fig. 9b, c). In addition, knockout of both $B R I P 1$ and $A L D H 2$ genes in the control cell lines SK-BR-3 and MDA-MB-468 led to a reduction in colony number comparable to the effect observed in the dependency cell lines, whereas no difference was observed when only one of the targets was ablated (Fig. 5d). These data further confirmed that the phenotypic effects observed upon genetic inhibition of BRIP1 are dependent on $A L D H 2$ levels.

Both BRIP1 and ALDH2 are involved in protecting cells from ACE-induced DNA damage, resulting in DNA double-strand breaks (DSBs) if not properly resolved. We hypothesized that inhibiting both protective axes could lead to an unsustainable increase in DSB formation, explaining the observed phenotypic effect. Of note, except for SK-BR-3, the tested cancer cell lines are characterized by heterozygous mutations with unknown impact in genes encoding different components of the FA pathway (Supplementary Fig. 9d). Therefore, to directly test the interaction of the ALDH2- BRIP1 dependency without potential confounding factors due to the cancer cell genetic background, we used the genetically stable RPE- ${ }^{\text {TPS3-I- }}$ cells as a model. We modeled ALDH2 deficiency by generating an $A L D H 2$ knock-out cell line (Supplementary Fig. 10a). To assess the effects of BRIP1 and potentially other components of the FA complex on the fitness of $A L D H 2 \mathrm{KO}$ cells in an unbiased manner, we performed a CRISPR-Cas9 screen using a custom designed pooled library that mainly targets DDR-related genes (Fig. 5e). In addition, we tested sensitivity of RPE-1 ${ }^{T P 53-/-}$ cells to exogenous ACE by performing these screens in the presence of $1 \mathrm{mM} \mathrm{ACE}$ (Fig. 5f). Consistent with the results from the cancer cell lines, targeting $B R I P 1$ as well as several other components of the FA pathway, such as FANCD2 and FANCI, significantly impaired the fitness of $A L D H 2 \mathrm{KO}$ cells as compared to the WT (Fig. 5e). These effects were exacerbated by the ACE treatment especially for BRIP1 (Fig. 5f). Consistently, and independent of the pooled screens, colony formation assays showed that simultaneous targeting of $A L D H 2$ and BRIP1 leads to a mild but significant decrease in the number of colonies (Supplementary Fig. 10b-d). Confluence analysis performed over a period of $72 \mathrm{~h}$ confirmed that cell proliferation was reduced in the double KO (Supplementary Fig. 10e). In addition, we tested the impact of single or combined KOs on the sensitivity of RPE-1 ${ }^{\text {TP53-I- }}$ cells to exogenous ACE on colony formation. BRIP1 $\mathrm{KO}$, but not $A L D H 2 \mathrm{KO}$, increased cell sensitivity to ACE and this effect was mildly elevated by the double KO (Supplementary Fig. 10f). These results indicate that BRIP1 is a major determinant of ACE sensitivity, consistent with the observation that the FA pathway mainly counteracts the genotoxic effects of ACE in mature, differentiated cells, while ALDH2 is dispensable [63].

Next, we explored the DSB response in RPE$1^{\text {TP53-I- }}$ cells upon single or combined KO. As proxy,

\footnotetext{
(See figure on next page.)

Fig. 5 Validation of the dependency of low-ALDH2 expressing cells on BRIP1 expression. a Western blot of BRIP1 and ALDH2 in the indicated breast cancer cell lines. The lower migrating band corresponds to ALDH2. RPE-1 = RPE-1 TP53-/- hTERT cells. Data are representative of 3 independent experiments. $\mathbf{b}$ Colony formation assay images of the indicated cell lines stably expressing Cas 9 and transfected with a scrambled gRNA or 2 independent gRNAs targeting BRIP1. Colonies were stained with crystal violet 15 days post-transfection. Images are representative of $\geq 3$ independent experiments. c Colony formation assay quantification in the control or dependency cell lines. The scrambled KO colony area is used for normalization. In the boxplots, centerlines mark the medians, box limits indicate the $25^{\text {th }}$ and $75^{\text {th }}$ percentiles, and whiskers extend to $5^{\text {th }}$ and $95^{\text {th }}$ percentiles. $P$-values are calculated based on Mann-Whitney $U$ test (** $p \leq 0.01$ ). d Colony formation assay quantification in MDA-MB-468 and SK-BR-3 stably expressing Cas9 and transfected with the indicated gRNAs. Bars represent normalized mean + standard deviation of 4 (SK-BR-3) or 3 (MDA-MB-468) independent experiments. The scrambled KO colony number is used for normalization. $P$-values are calculated with a one-way

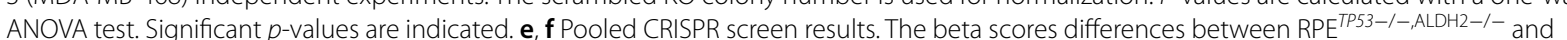
RPEE $^{\text {TP53-I- }}$ are showed as ranked plots, both in mock ( $\left.\mathbf{e}\right)$ and ACE $(\mathbf{f})$ treatments conditions. Gene names with a negative beta score difference 2.5 bigger than the standard deviation are showed in light blue, top 10 gene names with a positive beta score difference are shown in light red.

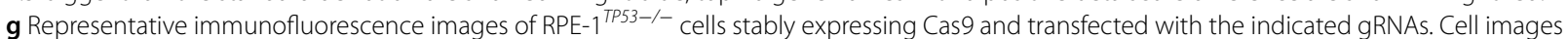
were acquired 6 days post-transfection. Nuclei are pseudocolored in gray; the yellow dots mark $\gamma$-H2AX foci. Scale bar $=20 \mu \mathrm{m}$. $\mathbf{h}$ Quantification of $\mathrm{Y}-\mathrm{H} 2 \mathrm{AX}$ foci formation in RPE-1 ${ }^{\text {TPS3-I- }}$ under the indicated conditions. Each dot indicates the number of foci/nucleus in each of the 4 biological replicates. $P$-values are calculated using a one-way ANOVA test. Selected significant $p$-values are indicated. The complete $p$-value list in provided in Additional file 4
} 
a

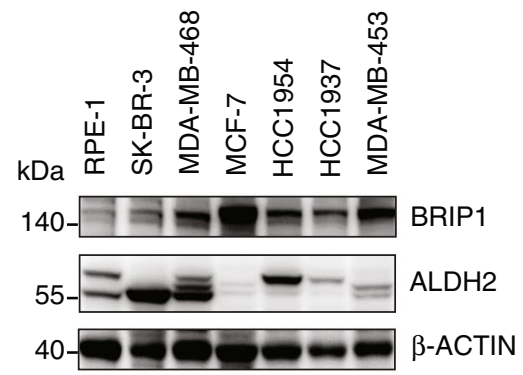

C $\frac{\text { gRNA }}{\text { BRIP1_guide1 BRIP1_guide2 }}$

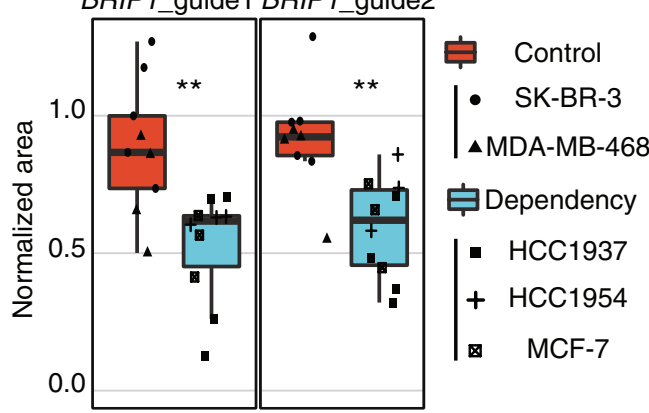

e

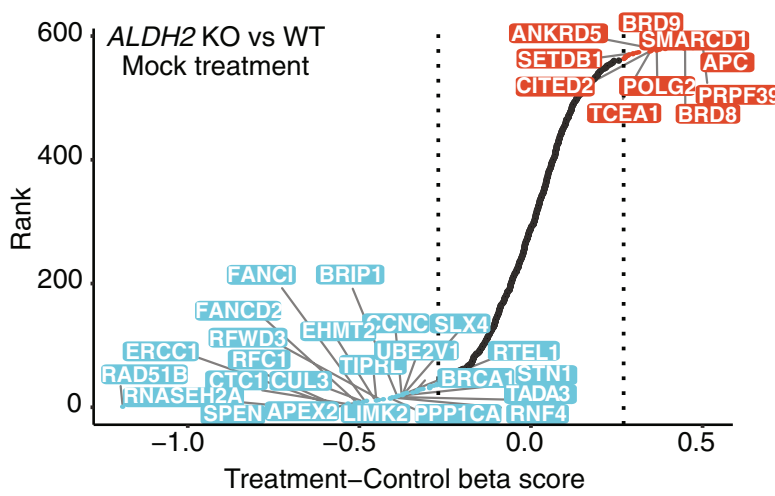

g

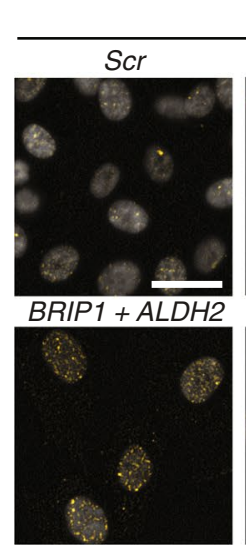

gRNA
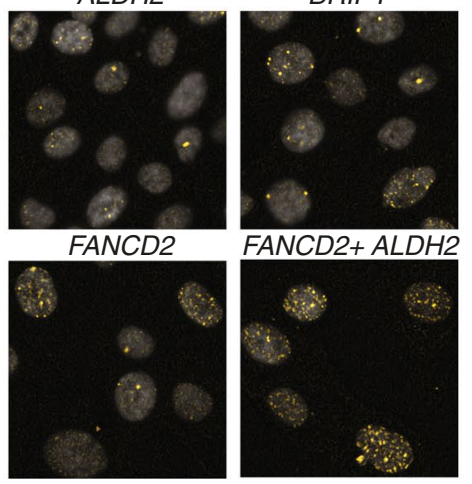

DNA $\gamma-\mathrm{H} 2 \mathrm{AX}$

b gRNA
Scr
BRIP1
guide1
BRIP1
guide2 Control Dependency

K-BR-3 MDA-MB- MCF-7 HCC1954 HCC1937
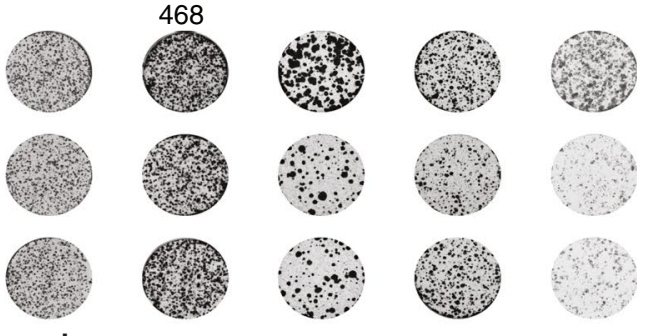

d

SK-BR-3
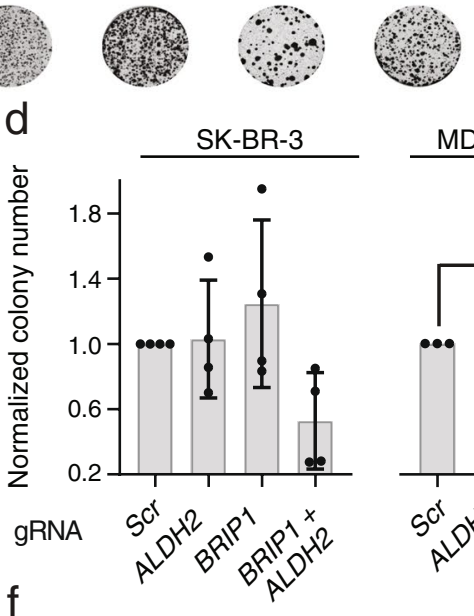

MDA-MB-468

f
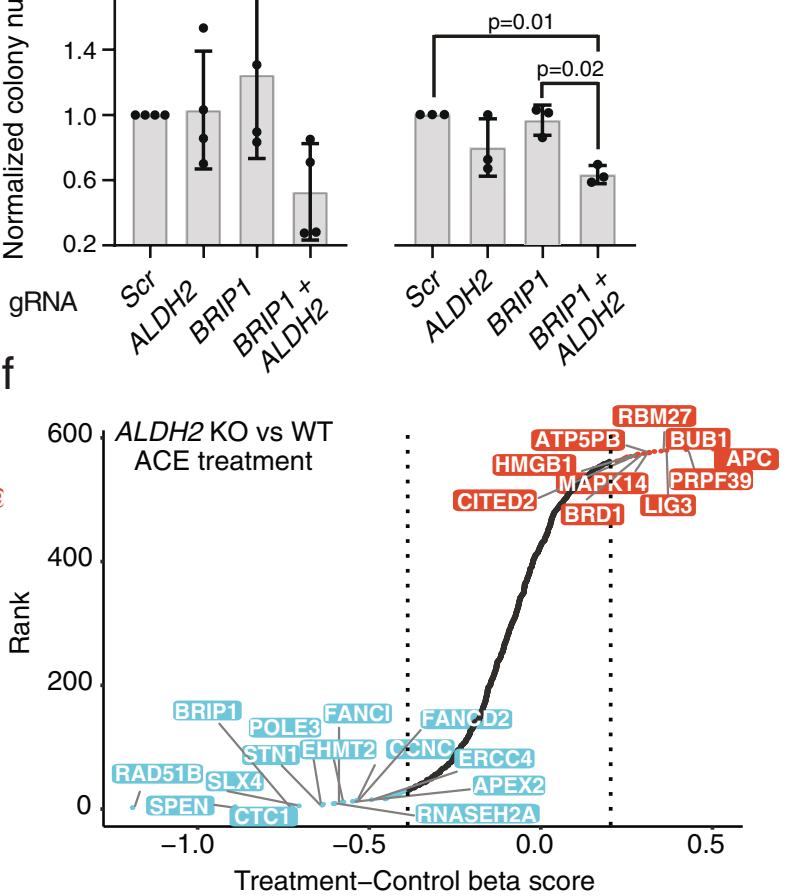

h

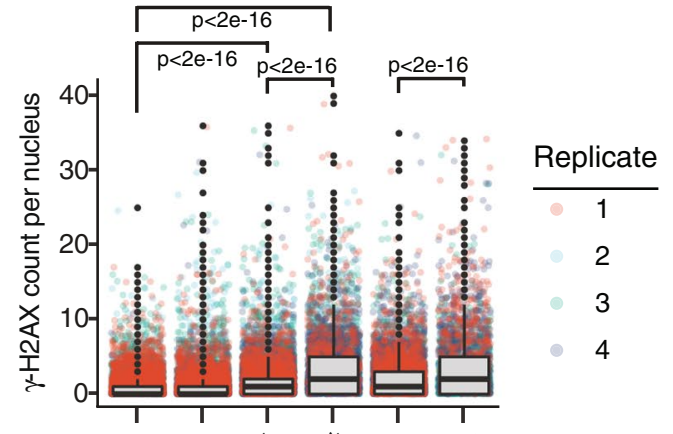

gRNA

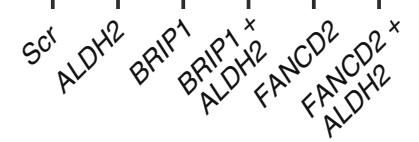

Fig. 5 (See legend on previous page.) 
we measured the nuclear foci formation of the histone variant H2AX phosphorylated at Ser139 ( $\gamma$-H2AX), a well-established marker of DNA damage. Consistent with our hypothesis, we detected increased nuclear foci count upon combined BRIP1 and ALDH2 KOs, compared to the single ones (Fig. $5 \mathrm{~g}, \mathrm{~h}$ ). In parallel, we tested how the cells respond to the KO of FANCD2, a component of the FA pathway whose function is reciprocally regulated by BRIP1 [67]. Similar to the effect observed upon BRIP1-ALDH2 KO, the combined $\mathrm{KO}$ of FANCD2 and $A L D H 2$ led to an increased formation of $\gamma-\mathrm{H} 2 \mathrm{AX}$ nuclear foci (Fig. 5g, h, Additional file 4). Consistent with the increased DNA damage measured upon the double KOs, the number of cells significantly decreased under the same conditions (Supplementary Fig. 10 g). In agreement with the decrease in colony forming capacity upon exogenous ACE treatment in BRIP1 KO cells, we detected an increase in the number of DNA damage foci under the same conditions (Supplementary Fig. $10 \mathrm{~h}$, Additional file 4). In summary, our data demonstrate that $B R I P 1$ deletion in low-expressing $A L D H 2$ cells triggers a dependency, presumably through DNA damage response induced by endogenous ACE.

In summary, using machine learning PARIS predicts cancer dependencies in an unbiased and scalable manner. We exemplified the power of this approach by studying the vulnerabilities of DDR-related genes and identified and validated two previously uncharacterized SL interactions. Our approach extends the catalog of cancer vulnerabilities, and provides a simple, rapid and robust way of testing additional SL interactions.

\section{Discussion}

The increasing availability of multiple datasets combining the genetic makeup of the cancer cell lines with largescale perturbance screens presents new opportunities for uncovering cancer vulnerabilities. In recent years, several studies presented ways to integrate these data and predict potential SL interactions computationally [7-13]. In this work, we address synthetic lethality prediction as a feature selection problem. The RF algorithm, the core of PARIS, can capture non-linear relationships and provides a robust method suitable for datasets in which the number of features is higher than the number of observations. Importantly, the use of the Boruta algorithm provides a way to overcome the multi-comparison error and the multicollinearity and overall, reduces the effect of randomness. The presence of highly correlated features is common in high throughput gene expression data. For this reason, it can be challenging to disentangle the contributions of collinear/co-expressed genes with respect to the outcome, in this case the dependency scores obtained from the gene knockouts. In multiple regression models this could be translated into a reduction of significance of coefficients. Often, a subset of those "redundant" features is selected to maximize and/or reduce the complexity of the final model. Boruta, being an all-relevant RF feature selection method, in the presence of collinear features, all well-related with the outcome, selects all of them and assigns them importance scores. This prevents removal of potentially important predictors and generates rankbased scores, therefore presents an overall better understanding of the contribution of each feature in explaining the outcome.

We provided a comparison of two types of importance score methods: the Gini and the permutation raw. Although the permutation raw method has a much higher computational cost, it generated the most unbiased and stable results with all the tested datasets. In addition, we showed how the use of a traditional RF algorithm with the corrected Gini importance score method can be adequate in terms of computational time and reliability. We suggest, however, to employ the Boruta algorithm with the permutation raw importance score method for final validations.

Here, we first applied PARIS to discover cancer vulnerabilities among known DDR-related genes and then searched for new SL pairs in the entire genome. In addition to providing the most robust SL pairs based on the importance scores, we also built a shiny app that allows the user to easily browse through the precomputed synthetic lethality network and to set custom filters. One advantage of our approach is that the pipeline can be applied to any set of genes and theoretically, to all the genes for which the dependency scores, expression and mutational status are available. However, we note that expression data is a better predictor than mutation data to explain the dependencies from the CRISPR-Cas9 screens as previously reported [55]. Results from expression datasets showed strong consistencies using different RF algorithms and cohorts. Contrary, mutation data led to more variable results although known SL interactions were correctly selected with high scores. It is likely that mutation-based data suffer from the weak predictability of their functional impact.

Searching for SL interactions among known DDR and related genes, we identified a previously uncharacterized vulnerability of CDKN2A-deficient cells to TYMS depletion. A possible direct role of CDKN2A p16 on nucleotide synthesis regulation has been proposed [68]. Particularly, p16 knockdown was shown to activate mTORC1 and to increase nucleotide synthesis in an RB-independent manner. TYMS plays an essential role in the de novo thymidine nucleotide synthesis [69]. Because of its indispensable role, it is commonly targeted in combination with platinum-based drugs for the 
treatment of various cancers such as non-small cell lung cancer and mesothelioma [70, 71]. The most widely used agent, 5-fluorouracil (5-FU) has been used in the clinic for decades, however, concerns regarding its lack of sensitivity to TYMS led to the design of anti-folates such as RTX or PMX [39]. In this work, we used cancer cell lines from lung, breast, thyroid, and head and neck cancers that carry nonsense mutations or CDKN2A deletions. Depending on the tissue type and expression levels of CDKN2A, TYMS, and TYMP, cells responded to pharmacological TYMS inhibition to different extents. Resistance to PMX can occur through several mechanisms such as TYMS expression levels, or multidrug resistance genes [72-76]. It was reported that TYMP overexpression correlates with TYMS KO sensitivity in cell lines and antifolate treatment in triple negative breast cancer patients [77]. In addition, TYMP promotes tumor growth and metastasis by preventing apoptosis and inducing angiogenesis [78]. Our results suggest that CDKN2A status also contribute to the sensitivity of TYMS inhibition. Thus, we suggest that both TYMP expression and $C D K N 2 A$ status should be monitored in order to better predict PMX sensitivity. Indeed, using cancer cell lines to confirm the TYMS dependency predicted by PARIS, we discovered that the most robust effects can be observed in those lines where TYMP is expressed at high levels and $C D K N 2 A$ is mutated or deleted. We note, however, that these effects can be more complex, and tissue type specific. For instance, based on DepMap data we observed a significant cooperation between TYMS and CDKN2A in esophageal cancer cell lines. However, in lung tumors neither TYMP levels nor CDKN2A status was able to explain TYMS KO sensitivity. In the future it might be interesting to understand if additional players (e.g. other components of the thymidine salvage pathway) can contribute to TYMS sensitivity in these tumors. On the other hand, our results may help to better stratify patients affected by esophageal tumors for which PMX use has been tested in clinical trials however, only showing limited success [79]. With the growing understanding of anti-folate sensitivity, better stratification of patients that can benefit from existing TYMS inhibitors may improve therapeutic outcome.

Analysis of the dependency scores between the DDR gene cohort and the whole genome revealed a dependency on BRIP1 for cells expressing low $A L D H 2$ levels. Decreased $A L D H 2$ expression is commonly found in human tumors (http://gent2.appex.kr/gent2/) and is associated with poor cancer prognosis. Silencing of the $A L D H 2$ locus by DNA methylation has been reported as a mechanism of $A L D H 2$ downregulation in lung adenocarcinoma [80-82]. Consistently, we found $A L D H 2$ promoter is hypermethylated in the panel of low- $A L D H 2$ expressing breast cancer cell lines used in this study.

Upregulation of FA pathway genes is frequently observed in tumors and found to be associated with chemo-resistance [83]. Previous studies have demonstrated that genetic loss of FA pathway components exacerbates ACE-mediated genotoxicity in mouse hematopoietic stem cells $[62,63,65]$. These studies provided important evidence for understanding the impact of ACE-induced DNA damage and the protective role of ALDH2 in the context of FA, a genetic disease leading to bone marrow failure and developmental disorders. Here we show that loss of $A L D H 2$ induces a dependency on BRIP1. We observed mild but consistent effects in both cancer cell lines and genetically stable epithelial cells. While BRIP1 represented the highest confidence hit predicted by PARIS, analysis of human tumor samples highlighted a consistent upregulation of FA genes in a low- $A L D H 2$ expression background. Accordingly, the combined inhibition of FANCD2 and $A L D H 2$ in RPE-1 cells allowed us to recapitulate the same effects on decreased colony formation and elevated DNA damage that we observed upon BRIP1-ALDH2 KO. Therefore, our results help strengthen the connection between the function of the FA pathway and the role of ALDH2, also consistent with a recent study in Acute Myeloid Leukemia [84]. Overall, we suggest that in a cancer context, low $A L D H 2$ expression can be used as a parameter to predict treatment outcome of drugs targeting the FA pathway, which can be potentially developed for clinical use [8386]. Specifically, we propose that our results represent the basis for future investigations of the role of BRIP1 as a potential cancer therapeutic target in an in vivo setting. Although BRIP1 inhibitors are not currently available, there is increased recognition of the therapeutic role of DNA helicases [87], and a deeper understanding of individual helicases' structure and property may pave the way for the development of specific drugs. In conclusion, our results shed light on a cancer dependency that may help establishing personalized therapeutic approaches.

\section{Conclusions}

We present a computational pipeline to infer SL interactions and vulnerabilities in cancers based on RF feature selection algorithms. The main advantage of PARIS is the ability to select all the relevant features to explain the observed dependency after a gene knockout and to assign them an importance score. We demonstrated how this score is proportional to the likelihood of observing a strong gene interaction and, ultimately, a synthetic lethality. Beyond the main aim to retrieve "one to one" SL relationships, PARIS, due to its nature, can provide additional hints for cases in which multiple factors contribute 
to the increased sensitivity to a specific gene (e.g. TYMS and $W R N$ ), thus it can also uncover multifactorial vulnerabilities. While we illustrated PARIS' capacity to predict dozens of already well-studied SL interactions as well as to uncover previously undescribed ones, some aspects of our approach can be further improved in the future. For instance, a different implementation of the pipeline may reduce the computational runtime or additional perturbation screen data can be integrated for more robust analyses. Importantly, since the PARIS pipeline is easy to implement, updated versions can be continuously built thanks to the continuous and rapid expansion of available omics data and CRISPR/Cas9-based screens. This will become increasingly important to spot additional vulnerabilities in cases where more observations, i.e. cell lines carrying a particular deficiency, are required to obtain meaningful results. Overall, our approach offers an original and reliable solution to inference of vulnerabilities in cancer.

\section{Methods}

\section{PARIS bioinformatic pipeline}

The CRISPR-Cas9 screens dependency scores, expression and mutation data were downloaded from the DepMap consortium website (version DepMap19Q3). Single nucleotide variants pathogenicity was predicted with FATHMM-MKL and only mutations with a coding score higher than 0.7 or annotated as damaging or TCGA hotspots were labeled as pathogenic. The presence of a pathogenic mutated gene for each cell lines was coded like $0=$ not mutated and $1=$ mutated. RNAseq $\log 2(\mathrm{TPM}+1)$ gene expression for protein coding genes was used.

In order to select mutated genes and gene expression explaining the gene dependency across all the cell lines, a RF-based feature selection step was performed. Particularly, for each gene in the CRISPR-Cas9 screen the Boruta algorithm was run four times, using alternatively the Gini or the raw permutation importance scores and the mutation or the expression data as independent variables. The maximum number of iterations of the Boruta algorithm was set to 500. As a third feature selection approach, a RF algorithm was used with the corrected impurity (corrected Gini) as importance score.

In each loop Boruta assigns a hit $(+1)$ to each feature showing an importance score higher than shadowMax. For each feature, hits are counted until they become either significantly higher or lower than what expected by random attempts (estimated as the cumulative distribution function of a binomial distribution with a probability of success by a chance of 0.5 ). In the first case, the feature is confirmed and in the second one is rejected and removed. The $p$-values are adjusted with the Bonferroni method and a $p$-value cut-off of 0.01 is used. Non-significant features that are neither confirmed nor rejected, are tested again in the next iteration.

The features selected as important to explain the dependency score were extracted and the Pearson correlation coefficient was calculated for each pair of genes to understand the direction of the relationship. Positive correlations were selected from the mutation-dependency pairs and negative correlation from the expressiondependency one. Self-pairs were also removed.

The importance scores were scaled by group between 0 and 1 , where 1 was the higher score in the group.

The pipeline was run on a 64 bit Ubuntu (version 16.04) system, with Intel ${ }^{\circledR}$ Xeon ${ }^{\circledR}$ Gold 6152 CPU @ $2.10 \mathrm{GHz}$ and 1.47 TB memory. For the DDR vs DDR cohort, the computational time for each gene model was $\sim 2$ min with the permutation raw as importance score method and $\sim 15 \mathrm{~s}$ with the Gini one. For the DDR vs ALL cohort, each gene model required $\sim 15$ min with the permutation raw and $\sim 3$ min with the Gini method. The total time needed to complete the entire DDR vs ALL cohort (4 models for each gene; expression/mutation and two importance score methods) was $\sim 13$ days.

Except for FATHMM-MKL, all the steps were performed in R. Boruta (version 7.0.0) and ranger (version 0.12.1) packages were used for the RF feature selection step.

\section{Analysis of gene pairs}

The scaled importance scores of the selected gene pairs were plotted as a density distribution and as a histogram grouped by scoring methods (Gini, permutation raw and corrected Gini) and features cohorts (expression or mutation). To set a threshold of confidence, we proceeded as follow. The head/tail breaks algorithm is specifically applied to cluster long tailed distributions. Briefly, the data values, the scaled importance scores in this case, are divided into greater (head) or less (tail) than the mean and the first subset is used as next distribution and the new mean is computed. The process is recursively applied until the head is not a minority of the distribution anymore (length (head)/length (tail) $<=40 \%$ ). This last break point is used as a threshold to identify high-confidence pairs.

In order to compare the different importance scores methods only the gene pairs selected by all the three approaches were used in the next analysis.

Each selected gene pair was labeled as "high-confidence" if its scaled importance score was above the threshold for any of the methods and as "low-confidence" if its scaled importance score was below the threshold for all of the methods. 
For each selected gene pair, the combined score from STRING database was retrieved if the interaction between the two genes was available. The frequency of interacting genes over the total selected in the different groups was also calculated.

Potential SL interactions were prioritized as follows: gene pairs above the mentioned cutoff were ranked based on their scaled raw permutation importance score. Additionally, genes with a coefficient of variation of the dependency score distribution lower than 0.3 were considered essential and filtered out.

\section{R shiny app}

The selected gene pairs out from the feature selection step were saved into a.csv file reporting: the two gene names, the Pearson correlation coefficient, the importance score from the RF feature selection, the importance score methods and the features source as group and the scaled importance score. A.csv file containing the gene name, the median, coefficient of variation, standard deviation and range of the dependency scores of all screened genes was also generated.

An $\mathrm{R}$ shiny app was built to allow easy visualization. The data were represented as an interactive directed graph in which each node represent a gene and each edge a relationship; the arrow starts from the mutated or dysregulated gene and arrives to the dependent gene. The color of the edge indicates the source of the evidence (mutation or expression) whereas the width is proportional to the absolute value of the Pearson correlation coefficient. The color of the node shows the median of the dependency score of the gene in a grey scale to easily identify essential genes.

The shiny app allows users to filter the gene pairs selecting one or both the two feature cohorts (mutation and/or expression) and applying independent thresholds for them, selecting one of the importance score methods (Gini, permutation raw, corrected Gini) and applying the filtering step based on the Pearson correlation coefficient (only negative correlation for expression and only positive correlation for mutation). The network is redrawn in real-time accordingly to the selected filters and can be exported as a.png file. Cytoscape-compatible network and nodes.csv files can be also downloaded.

The filtering step based on the direction of the relationship can be also bypassed to investigate all the possibilities, e.g. to investigate the dependencies in the case of oncogenic expression.

The $\mathrm{R}$ shiny app was developed in $\mathrm{R}$ using the shiny (version 1.5.0) and visNetwork (version 2.0.9) packages.

\section{TCGA and GTEX data analysis}

TCGA and GTEX BRIP1 and ALDH2 expression data were retrieved from the Xena browser (https://xenab rowser.net/). Correlation was calculated using the Pearson coefficient.

\section{TCGA Differential gene expression analysis}

TCGA data were retrieved using the TCGAbiolinks $R$ package (version 2.14.1). Samples were ranked based on their $A L D H 2$ expression level. The top and bottom $2 \%$ of the samples were used as high and low expressing $A L D H 2$ groups. The differential gene expression analysis was performed with DESeq2 (version 1.26.0). Genes with an adjusted pvalue $<0.01$ were considered to be differentially expressed. EnrichR (version 2.1) was used for the enrichment analysis.

\section{Cell lines and cell culture}

Cell lines were maintained in a humified incubator at $37{ }^{\circ} \mathrm{C}, 5 \% \mathrm{CO}_{2}$. A table of the cell lines used in this study and their culturing media is listed in Supplementary Table 1.

\section{Generation of stable cell lines Cas9-positive cell lines}

A table of cell lines and sources used in this study is listed in Supplementary Table 1 or detailed in [88]. Briefly, cells were infected using hEF1 $\alpha$-TurboGFP-Cas 9 Nuclease viral particles (Dharmacon VCAS11864), according to manufacturer's specifications. GFP-positive cells were sorted using FACS Aria II instrument (BD Biosciences). Alternatively, cells were infected using InduciblehEF1 $\alpha$-Blast-Cas9 Nuclease viral particles (Dharmacon VCAS11864), according to manufacturer's specifications and selected with blasticidin.

\section{TYMP-positive cell lines}

Cells were infected with lentiviral particles expressing TYMP-GFP (Twist Biosciences - Supplementary Data). GFP-positive cells were sorted using FACS Aria II instrument (BD Biosciences).

\section{ALDH2 and CDKN2A KO}

RPE-1 ${ }^{\text {TP53-I- }}$ cells stably expressing an inducible Cas9 transgene were transfected with a gRNA targeting ALDH2 or CDKN2A (sequence provided in the Supplementary Table 2), in the presence of $1 \mu \mathrm{g} / \mathrm{ml}$ doxycycline. Three days post transfection cells were seeded on 96-well plates for single clone formation. The KO efficiency of selected single cell-derived clones was assessed by Western blot. Two validated KO clones were mixed and used for downstream experiments. 


\section{C-MYC-positive cell lines}

RPE-1 ${ }^{\text {TP53-I- }}$ or RPE-1 ${ }^{\text {TP53-I- CDKN2A-/- Cells were }}$ infected with lentiviral particles expressing cMYC-BFP (Twist Biosciences - Supplementary Data). BFP-positive cells were sorted using FACS Aria II instrument (BD Biosciences).

\section{Lentivirus generation}

Lentiviral plasmids pMD2G (12,259) and psPAX2 $(12,260)$ were obtained from Addgene. A lentiviral plasmid expressing TYMP cDNA was purchased from Twist Biosciences. One $\mu \mathrm{g}$ of each plasmid was transfected to 293FT cells using Lipofectamine 3000 according to manufacturers' protocol. Viral particles were collected $72 \mathrm{~h}$ post transfection, filtered using a $0.45 \mu \mathrm{m}$ low protein binding membrane Steriflip HV/PVDF (Millipore) and stored at $-80^{\circ} \mathrm{C}$.

\section{Transfection of gRNAs}

Exponentially proliferating cells at $70-80 \%$ confluency were transfected with 2.5 pmol gRNA complexes and $0.3 \mu \mathrm{l}$ of Lipofectamine RNAiMAX/96 well (Invitrogen $13,778,150)$ according to the manufacturer's protocols. A detailed list of gRNAs is provided in the Supplementary Table 2.

\section{Pooled CRISPR screens}

Custom designed lentiviral library was generated by Cellecta. Lentiviral particles were produced by co-transfecting 293FT cells with $50 \mu \mathrm{g}$ of custom designed library (Cellecta), $50 \mu \mathrm{g}$ of pMD2.G (Addgene, 12,259), and $75 \mu \mathrm{g}$ of psPAX2 (Addgene, 12,260) using Lipofectamine 3000 (Thermo Fisher Scientific, L3000001) following manufacturer's specifications. After $60 \mathrm{~h}$, viral supernatant was collected and filtered through a $0.45 \mu \mathrm{m}$ low protein binding membrane Steriflip HV/PVDF (Millipore).

Ten million $\mathrm{RPE}^{\text {TP53-I- }}$ and $\mathrm{RPE}^{\text {TP53-I-,ALDH2-l- }}$ cells per library and per replicate, aliquoted to 600.000 cells per well of a 6-well plate, were spinfected with the lentiviral library at 2,000 rpm, for $2 \mathrm{~h}$ at $37{ }^{\circ} \mathrm{C}$, with the addition of $8 \mu \mathrm{g} / \mathrm{mL}$ polybrene (Sigma-Aldrich, TR-1003) to reach a multiplicity of infection of 0.3 . Cells were left to recover in fresh medium for $24 \mathrm{~h}$ incubated at $37^{\circ} \mathrm{C}, 5 \% \mathrm{CO}_{2}$. The infected cells were selected with $10 \mu \mathrm{g} / \mathrm{ml}$ puromycin (Thermo Fisher Scientific, A1113803) for 3 days. Following puromycin selection, Cas9 was induced with $1 \mu \mathrm{g} / \mathrm{ml}$ Doxycycline (Day0). Cells were incubated in a humidified atmosphere to proliferate for a total of 21 days and subdivided to 2 million cells every three days to maintain appropriate coverage. ACE treatment was started after 6 days of Doxycycline induction by addition of $1 \mathrm{mM}$ ACE and kept throughout the experiment by replenishing the ACE every time the cells were subdivided.
For preparation of the sequencing libraries, genomic DNA was isolated from the initial and final time points using QIAamp DNA Blood Kit (Qiagen, 51,106) following manufacturer's protocol. Eight $\mu \mathrm{g}$ DNA was used as a template in two separate reactions of 50ul for 12 cycles using plasmid-specific primers for the amplification of the sgRNA coding sequences. An additional round of PCR amplification was run for 10 cycles for the addition of Illumina adaptors and sample specific barcodes. Q5 NEBNext Hot Start polymerase master mix (M0543) was used for the PCR reactions according to manufacturer's specifications. The primers used for the reactions are depicted in the Supplementary Table 2. PCR reaction products of 390 bps were purified using 0.8X Agencourt AMPure XP (Beckman Coulter, A63880) and eluted in low EDTA TE buffer (Thermo Fisher, 12,090,015). All samples were pooled and sequenced using Illumina NextSeq platform (Illumina), using 75 base single reads.

The cell fitness is calculated based on the gRNA counts at the beginning and at the end of the experiment since the selection process may enrich or deplete sgRNAs from the cell population. Accordingly, the gRNAs that result in loss of cell fitness are expected to be depleted from the cell population at the final time point as compared to the first time point. To count the number of gRNAs in each library, and to perform downstream analyses we used MEMcrispR [89] that enables efficient analysis of genome-wide count-based screens based on linear mixed-effects regression on the initial and final timepoints. For each sample, the sgRNAs were aligned and the number of gRNAs were normalized according to sequencing depth. Afterwards, comparing the Day 6 and Day 21 of each sample, the $P$-values and beta scores that measure the degree of the perturbation effect were calculated for each gene using the mixed effect model. Finally, the beta scores differences between RPE ${ }^{T P 53-l-, A L D H 2-/-}$ and $\mathrm{RPE}^{T P 53-1-}$ were calculated for each gene. Genes with a positive or a negative beta score differences enhance or decrease the $\mathrm{RPE}^{T P 53-/-, \mathrm{ALDH} 2-/-}$ growth compared to RPE ${ }^{T P 53-/-}$ after being knocked out, respectively. The RankView function from the MAGeCKFlute $\mathrm{R}$ package was used to plot the results.

\section{Cell viability assays}

CellTiter-Glo (Promega) was used to determine cell viability, according to the manufacturer's protocol. Cells were seeded on a 96-well white plate with a clear flat bottom (Costart ${ }^{\circledR}$ Assay plate, Corning). The GloMax-Multi detection system (Promega) was used as a luminometer to quantify the presence of ATP as an indicator of metabolically active cells. 
For thymidine rescue experiments, CellTiter-Fluor (Promega) was used according to manufacturer's protocol in parallel to CellTiter-Glo experiments as described above.

IC50 values were calculated with a non-linear regression model using GraphPad PRISM.

\section{Colony formation assay}

Three days post gRNA transfection, equal number of cells were plated in 6-well plates. After 12-15 days colonies were stained with $0,2 \%$ crystal violet (Sigma-Aldrich V5265) and scanned with a GelCount Scanner (Oxford Optronix). The colony number was quantified using the ImageJ software either by manual counting with a multipoint tool or using a colony area plugin [90].

\section{RNA extraction, cDNA synthesis and RT-qPCR}

RNA was isolated using RNeasy Plus Mini kit (Qiagen), according to manufacturer's instructions. cDNA synthesis was performed using the qScript cDNA Synthesis Kit (VWR International). RT-qPCR was carried out using QuantStudio 3 Real-Time PCR System, following manufacturer's instructions. The oligonucleotide list is presented in Reagents and Tools Table. 36B4 was used as housekeeping gene for internal normalization.

\section{Western blot}

Cells were collected in ice-cold PBS. Cell pellets were lysed in RIPA buffer (Cell Signaling, 9806S) supplemented with 0,2\% SDS, PMSF and protease/phosphatase inhibitor cocktail (Cell Signaling, 9806S). Twenty $\mu \mathrm{g}$ of whole cell lysates was resolved on NuPAGE 4-12\% BisTris Protein Gels (Invitrogen) and transferred onto PVDF membranes (neolab Migge GmbH, IPFL00010). Membranes were blocked in 5\% BSA and incubated overnight with primary antibodies (Reagents and Tools Table). Anti-mouse or anti-Rabbit IgG, HRP-linked Antibody (Cell signaling, 7076; 7074) were used as secondary antibodies and signals were detected using Vilber FUSION FX7 (Vilber Lourmat). VINCULIN and $\beta$-ACTIN served as loading controls.

\section{Immunofluorescence}

Cells were seeded in 96 well glass bottom plates (Cellvis, P96-1.5H-N), fixed for 10 min with 4\% PFA at room temperature, washed 3 times with D-PBS, permeabilized for 10 min with $0,25 \%$ Triton-X-100 in D-PBS and blocked with $10 \%$ FBS $-0.1 \%$ Triton-X100 for $1 \mathrm{~h}$ at room temperature. Cells were then stained overnight at 4 degrees with $\gamma$-H2AX primary antibody (Merck Millipore 05-636) diluted 1:500 in 3\% BSA/D-PBS, washed 3 times with D-PBS and stained with secondary antibody (Alexa Fluor-594, goat anti mouse, 1:1000) and Hoechst (1:4000) in $3 \% \mathrm{BSA} / \mathrm{D}-\mathrm{PBS}$ for $1 \mathrm{~h}$. Cells were stored in the dark at $4{ }^{\circ} \mathrm{C}$ until observed under the microscope.

\section{Microscopy}

Fluorescent images were acquired on a Nikon Ti-E automated epifluorescent microscope. The microscope was equipped with a DS-Qi2 camera and a Sola nIR LED lamp. The filter sets were provided by Nikon for DAPI (DAPI-A-2360A), Alexa Fluor 568 (Cy3-A-4040C)) and Alexa Fluor 647 (LED-Cy5-5070A). The objectives used were P-Fluor 40X, numerical aperture 0.60 (Nikon). We used a hardware base autofocusing system called the perfect focus system (PFS) from Nikon to automatically focus the cells on the field of view. For each replicate, we imaged 2 wells per condition with multiple images (a minimum of 4 and a maximum of 9) per well.

For quantification of $\gamma-\mathrm{H} 2 \mathrm{AX}$ foci, and CyclinA levels, images were analyzed by ImageJ with custom made macros. Briefly, the nuclei were segmented from DAPI channel image by automated thresholding and watershed procedure to split touching nuclei. For analysis of CyclinA levels, the mean intensities of the nuclear CyclinA were measured based on the segmented nuclei in each image.

For counting $\gamma-\mathrm{H} 2 \mathrm{AX}$ foci, similar nuclear segmentation steps were applied. Based on the segmented nuclei in each image then we applied another round of thresholding and segmentation for $\gamma-\mathrm{H} 2 \mathrm{AX}$ channel. Downstream analyses of the foci counts were performed in $R$.

\section{Live cell imaging}

Three days post gRNA transfection, equal number of cells were plated in 96-well plates. Cell proliferation was monitored over time using the Incucyte S3 scanner. Phase contrast images were acquired every $12 \mathrm{~h}$ using a $10 \times$ objective and analyzed using the Incucyte S3 LiveCell Analysis System.

\section{Statistical analyses}

The applied statistical tests are specified in each figure legend.

\section{Abbreviations}

PARIS: Pan-Cancer inferred synthetic lethalities; SL: Synthetic lethal; RF: Random forest; CCLE: Cancer Cell Line Encyclopedia; TCGA: The cancer genome atlas; GTEX: Genotype-tissue expression; PMX: Pemetrexed; dUMP: Deoxyuridine monophosphate; dTMP: Deoxythymidine monophosphate; FA: Fanconi anemia; ICL: Interstrand crosslink; ACE: Acetaldehyde; DSBs: DNA doublestrand breaks; RPE-1: Retinal pigment epithelisal; KO: Knock out. 


\section{Supplementary Information}

The online version contains supplementary material available at https://doi. org/10.1186/s12943-021-01405-8.

\section{Additional file 1. \\ Additional file 2. \\ Additional file 3. \\ Additional file 4. \\ Additional file 5 .}

employees of BioMed X Institute $(\mathrm{GmbH})$, Heidelberg, Germany. Amir Abdollahi is an academic mentor at BioMed $X$ Institute and receives research grants from Merck KGaA.

\section{Author details}

${ }^{1}$ BioMed X Institute (GmbH), Im Neuenheimer Feld 583, 69120 Heidelberg, Germany. ${ }^{2}$ Division of Molecular and Translational Radiation Oncology, National Centre for Tumour Diseases (NCT), Heidelberg University Hospital, 69120 Heidelberg, Germany. ${ }^{3}$ Translational Innovation Platform Oncology \& Immuno-Oncology, Merck KGaA, Frankfurter Str. 250, 64293 Darmstadt, Germany.

Received: 1 April 2021 Accepted: 10 August 2021

Published online: 28 August 2021

\section{Authors' information}

Salvatore Benfatto, Özdemirhan Serçin, Francesca R. Dejure contributed equally to this work.

\section{Acknowledgements}

We thank Vladimir Benes from EMBL GeneCore for assistance with sequencing. We thank Holger Lorenz from ZMBH, Heidelberg for developing the ImageJ macros used for image analyses. We would also like to thank Ashley Sanders (MDC, Berlin) and Thomas Rückle (BioMed X Institute) for critical comments on the manuscript.

\section{Authors' contributions}

B.R.M. and Ö.S. conceived the idea and B.R.M. designed the study. S.B. developed the computational algorithms for predicting SL interactions and carried out all the bioinformatic analyses. Ö.S. carried out experiments related to Fig. 3. F.R.D. carried out experiments related to Fig. 5. A.A. and F.T.Z provided critical input and materials. B.R.M., S.B., F.R.D. and Ö.S. wrote the paper with input from all authors. The authors read and approved the final manuscript.

\section{Funding}

This work was supported by Merck KGaA, Darmstadt, Germany (CrossRef Funder ID: 10.13039/100009945).

\section{Availability of data and materials}

All data associated with this study are presented in the paper and DepMap data were downloaded from https://depmap.org/ (version DepMap19Q3). The R shiny apps showing the preprocessed PARIS results can be downloaded at https://github.com/sbenfatto/PARIS_DDR_vs_DDR and at https://github. com/sbenfatto/PARIS_DDR_vs_ALL and run on a local computer. They are also freely usable at https://sbenfatto.shinyapps.io/paris_ddr_vs_ddr/ and https:// sbenfatto.shinyapps.io/paris_ddr_vs_all/.

Source data underlying Fig. 2 is provided as Additional file 1. Source data underlying Fig. $3 \mathrm{~d}$ is provided as source Additional file 2. Source data underlying Fig. 4 is provided as Additional file 3.

Source data underlying Fig. 5h and Supplementary Figs. $10 \mathrm{~g}$ and h are provided as Additional file 4.

The PARIS pipeline repository is available at https://github.com/sbenfatto/ PARIS, it includes the main functions to run PARIS as well as the ones used to perform the analysis and to generate the plots in the present work. Correspondence and requests for materials should be addressed to B.R.M (mardin@bio.mx).

\section{Declarations}

\section{Ethics approval and consent to participate}

Not applicable.

\section{Consent for publication}

All authors gave consent for the publication of this study.

\section{Competing interests}

FrankT. Zenke is an employee of Merck KGaA, Darmstadt, Germany. Salvatore Benfatto, Francesca R. Dejure, Özdemirhan Serçin, and Balca R. Mardin are

\section{References}

1. Nijman, S. M. B. Synthetic lethality: General principles, utility and detection using genetic screens in human cells. FEBS Letters. 2011.https://doi. org/10.1016/j.febslet.2010.11.024.

2. Bridges, C. B. The Origin of variations in Sexual and Sex-Limited characters. Am Nat. 1922.https://doi.org/10.1086/279847.

3. Dobzhansky, T. Genetics of natural populations; recombination and variability in populations of Drosophila pseudoobscura. Genetics. 1946;31(3):269-90.

4. O'Neil, N. J., Bailey, M. L. \& Hieter, P. Synthetic lethality and cancer. Nat Rev Genet. 2017.https://doi.org/10.1038/nrg.2017.47.

5. Lord, C. J. \& Ashworth, A. PARP inhibitors: Synthetic lethality in the clinic. Science. 2017.https://doi.org/10.1126/science.aam7344.

6. Tsherniak, A. et al. Defining a cancer dependency map. Cell. 2017.https:// doi.org/10.1016/j.cell.2017.06.010.

7. Jerby-Arnon $L$, et al. Predicting cancer-specific vulnerability via datadriven detection of synthetic lethality. Cell. 2014;158:1199-209.

8. Srihari S, Singla J, Wong $L$ \& Ragan MA. Inferring synthetic lethal interactions from mutual exclusivity of genetic events in cancer. Biol Direct. 2015.https://doi.org/10.1186/s13062-015-0086-1.

9. Sinha S, et al. Systematic discovery of mutation-specific synthetic lethals by mining pan-cancer human primary tumor data. Nat Commun. 2017:8:1-13.

10. Apaolaza l, et al. An in-silico approach to predict and exploit synthetic lethality in cancer metabolism. Nat Commun. 2017;8:1-9.

11. Lee JS, et al. Harnessing synthetic lethality to predict the response to cancer treatment. Nat Commun. 2018;9:1-12.

12. Das S, Deng X, Camphausen K, Shankavaram U, Schwartz R. DiscoverSL: An $R$ package for multi-omic data driven prediction of synthetic lethality in cancers. Bioinformatics. 2019;35:701-2.

13. Li JR, et al. Identification of synthetic lethality based on a functional network by using machine learning algorithms. J Cell Biochem. 2019:120:405-16.

14. Behan FM, et al. Prioritization of cancer therapeutic targets using CRISPRCas9 screens. Nature. 2019;568:511-6.

15. Kategaya L, Perumal SK, Hager JH, Belmont LD. Werner syndrome helicase is required for the survival of cancer cells with microsatellite instability. iscience. 2019;13:488-97.

16. Lieb $\mathrm{S}$, et al. Werner syndrome helicase is a selective vulnerability of microsatellite instability- high tumor cells. Elife. 2019;8:1-22.

17. Chan EM, et al. WRN helicase is a synthetic lethal target in microsatellite unstable cancers. Nature. 2019;568:551-6.

18. Nilsson R, Peña JM, Björkegren J. \& Tegnér J. Consistent feature selection for pattern recognition in polynomial time. J Mach Learn. Res. 2007;8(21):589-612.

19. Saeys Y, Inza I. \& Larrañaga P. A review of feature selection techniques in bioinformatics. Bioinformatics. 2007.https://doi.org/10.1093/bioinforma tics/btm344.

20. Kursa MB. Robustness of Random Forest-based gene selection methods. BMC Bioinformatics. 2014. https://doi.org/10.1186/1471-2105-15-8.

21. Meyers RM. et al. Computational correction of copy number effect improves specificity of CRISPR-Cas 9 essentiality screens in cancer cells. Nat Genet. 2017.https://doi.org/10.1038/ng.3984. 
22. Shihab HA. et al.An integrative approach to predicting the functional effects of non-coding and coding sequence variation. Bioinformatics.2015.https://doi.org/10.1093/bioinformatics/btv009.

23. Kursa MB. \& Rudnicki WR. Feature selection with the boruta package. J Stat Softw. 2010;36

24. Degenhardt F, Seifert S \& Szymczak S. Evaluation of variable selection methods for random forests and omics data sets. Brief Bioinform. 2019.https://doi.org/10.1093/bib/bbx124.

25. Pearl LH, Schierz AC, Ward SE, Al-Lazikani B, Pearl FMG. Therapeutic opportunities within the DNA damage response. Nat Rev Cancer. 2015.https://doi.org/10.1038/nrc3891.

26. Roidos P. et al.A scalable CRISPR/Cas9-based fluorescent reporter assay to study DNA double-strand break repair choice. Nat Commun. 2020.https://doi.org/10.1038/s41467-020-17962-3.

27. Kelso TWR. et al. Chromatin accessibility underlies synthetic lethality of SWI/SNF subunits in ARID1A-mutant cancers. Elife. 2017.https://doi.org/ 10.7554/eLife.30506.001.

28. Helming KC. et al. ARID1B is a specific vulnerability in ARID1A-mutant cancers. Nat Med. 2014. https://doi.org/10.1038/nm.3480.

29. Nembrini S, König IR, Wright MN. The revival of the Gini importance? Bioinformatics. 2018;34:3711-8.

30. Franceschini, A. et al. STRING v9.1: Protein-protein interaction networks, with increased coverage and integration. Nucleic Acids Res. 2013. https:// doi.org/10.1093/nar/gks1094.

31. Chang W, Cheng J, Allaire J, Xie Y \& J, M. Shiny: Web Application Framework for R Shiny: Web Application Framework for R. (2020).

32. de Kegel B \& Ryan, CJ. Paralog buffering contributes to the variable essentiality of genes in cancer cell lines. PLoS Genet. 2019.https://doi.org/ 10.1371/journal.pgen.1008466.

33. Hoffman GR. et al. Functional epigenetics approach identifies BRM/ SMARCA2 as a critical synthetic lethal target in BRG1-deficient cancers. Proc Natl Acad Sci USA. 2014;111(8):3128-33. https://doi.org/10.1073/ pnas.131679311.

34. Karnezis AN. et al. Dual loss of the SWI/SNF complex ATPases SMARCA4/ BRG1 and SMARCA2/BRM is highly sensitive and specific for small cell carcinoma of the ovary, hypercalcaemic type. J Pathol. 2016. https://doi. org/10.1002/path.4633.

35. Guerrero-Martínez, JA. \& Reyes JC. High expression of SMARCA4 or SMARCA2 is frequently associated with an opposite prognosis in cancer Sci Rep. 2018.https://doi.org/10.1038/s41598-018-20217-3.

36. van der Lelij, P. et al. Synthetic lethality between the cohesin subunits STAG1 and STAG2 in diverse cancer contexts. Elife. 2017.https://doi.org/ 10.7554/eLife.26980.

37. Reis GF, et al. CDKN2A Loss Is Associated with Shortened Overall Survival in Lower-Grade (World Health Organization Grades II-III) Astrocytomas. J Neuropathol Exp Neurol. 2015;74:442-52.

38. Berger FG, Berger SH. Thymidylate synthase as a chemotherapeutic drug target: Where are we after fifty years? Cancer Biol Ther. 2006. https://doi. org/10.4161/cbt.5.9.3414.

39. Chattopadhyay S, Moran RG. \& Goldman ID. Pemetrexed: Biochemical and cellular pharmacology, mechanisms, and clinical applications. Mol Cancer Therapeutics. 2007.https://doi.org/10.1158/1535-7163. MCT-06-0343.

40. Visentin M, Zhao R, Goldman ID. The antifolates. Hematol/Oncol Clin North Am. 2012.https://doi.org/10.1016/j.hoc.2012.02.002.

41. Hou J. et al. Expression profiling-based subtyping identifies novel nonsmall cell lung cancer subgroups and implicates putative resistance to pemetrexed therapy. J Thorac Oncol. 2012.https://doi.org/10.1097/JTO. Ob013e3182352a45.

42. Obata T, Tanaka M, Suzuki Y, Sasaki T. The role of thymidylate synthase in pemetrexed-resistant malignant pleural mesothelioma cells. J Cancer Ther. 2013.https://doi.org/10.4236/jct.2013.46119.

43. Mariani BD, Slate DL, Schimke RT. S phase-specific synthesis of dihydrofolate reductase in Chinese hamster ovary cells. Proc Natl Acad Sci U S A. 1981;78:4985-9.

44. Ligabue A, Marverti G, Liebl U, Myllykallio H. Transcriptional activation and cell cycle block are the keys for 5-fluorouracil induced up-regulation of human thymidylate synthase expression. PLoS One. 2012;7(10):e47318. https://doi.org/10.1371/journal.pone.0047318.

45. Sherley JL, Kelly TJ. Regulation of human thymidine kinase during the cell cycle. J Biol Chem. 1988;263:8350-8.
46. Bello $\sqcup$. Regulation of thymidine kinase synthesis in human cells. Exp Cell Res. 1974.https://doi.org/10.1016/0014-4827(74)90790-3.

47. McDonald ER. et al. Project DRIVE: A compendium of cancer dependencies and synthetic lethal relationships uncovered by large-scale, deep RNAi screening. Cell. 2017.https://doi.org/10.1016/j.cell.2017. 07.005 .

48. Iltzsch MH, El Ko'uni, MH. \& Cha S. Kinetic studies of thymidine phosphorylase from mouse liver. Biochemistry. 1985. https://doi.org/10.1021/ bi00345a011.

49. Liu F, Yang X, Geng M, Huang M. Targeting ERK, an Achilles' Heel of the MAPK pathway, in cancer therapy. Acta Pharm Sin B. 2018;8:552-62.

50. Klein ME, Kovatcheva M, Davis LE, Tap WD, Koff A. CDK4/6 Inhibitors: The Mechanism of Action May Not Be as Simple as Once Thought. Cancer Cell. 2018;34:9-20.

51. Han K, et al. Synergistic drug combinations for cancer identified in a CRISPR screen for pairwise genetic interactions. Nat Biotechnol. 2017;35:463-74.

52. Najm FJ, et al. Orthologous CRISPR-Cas9 enzymes for combinatorial genetic screens. Nat Biotechnol. 2018;36:179-89.

53. van Delft MF, et al. The $\mathrm{BH} 3$ mimetic $A B T-737$ targets selective $\mathrm{BCl}-2$ proteins and efficiently induces apoptosis via Bak/Bax if $\mathrm{Mcl}-1$ is neutralized. Cancer Cell. 2006;10:389-99.

54. DeWeirdt PC. et al. Genetic screens in isogenic mammalian cell lines without single cell cloning. Nat Commun. 2020;11(1):752. https://doi.org/ 10.1038/s41467-020-14620-6.

55. Dempster JM. et al. Gene expression has more power for predicting in vitro cancer cell vulnerabilities than genomics. bioRxiv. 2020. https:// doi.org/10.1101/2020.02.21.959627.

56. Chen CH, Ferreira JCB, Gross ER. \& Mochly-Rosen D. Targeting aldehyde dehydrogenase 2: New therapeutic opportunities. Physiol Rev. 2014.https://doi.org/10.1152/physrev.00017.2013.

57. Datta A, Brosh RM. Holding all the cards—how fanconi anemia proteins deal with replication stress and preserve genomic stability. Genes. 2019.https://doi.org/10.3390/genes10020170

58. Niraj J, Färkkilä A. \& D'Andrea AD. The fanconi anemia pathway in cancer. Ann Rev Cancer Biol. 2019.https://doi.org/10.1146/annurev-cance rbio-030617-050422.

59. Peana AT. et al. Mystic acetaldehyde: The never-ending story on alcoholism. Front Behav Neurosci. 2017.https://doi.org/10.3389/fnbeh.2017. 00081.

60. Lorenti Garcia C. et al. Relationship between DNA lesions, DNA repair and chromosomal damage induced by acetaldehyde. Mutat Res- Fundam Mol Mech Mutagen. 2009. https://doi.org/10.1016/j.mrfmmm.2008.11. 008.

61. Hodskinson MR. et al. Alcohol-derived DNA crosslinks are repaired by two distinct mechanisms. Nature. 2020.https://doi.org/10.1038/ s41586-020-2059-5.

62. Langevin F, Crossan GP, Rosado IV, Arends MJ. \& Patel KJ. Fancd2 counteracts the toxic effects of naturally produced aldehydes in mice. Nature. 2011;475(7354):53-8. https://doi.org/10.1038/nature10192.

63. Garaycoechea Jl. et al. Genotoxic consequences of endogenous aldehydes on mouse haematopoietic stem cell function. Nature. 2012;489(7417):571-5. https://doi.org/10.1038/nature11368.

64. Hira A, et al. Variant ALDH2 is associated with accelerated progression of bone marrow failure in Japanese Fanconi anemia patients. Blood. 2013;122:3206-9.

65. Garaycoechea Jl. et al. Alcohol and endogenous aldehydes damage chromosomes and mutate stem cells. Nature. 2018.https://doi.org/10. 1038/nature25154.

66. Neve RM. et al. A collection of breast cancer cell lines for the study of functionally distinct cancer subtypes. Cancer Cell. 2006.https://doi.org/ 10.1016/j.ccr.2006.10.008.

67. Clark DW, Tripathi K, Dorsman JC. \& Palle K. FANCJ protein is important for the stability of FANCD2/FANCI proteins and protects them from proteasome and caspase-3 dependent degradation. Oncotarget. 2015.https:// doi.org/10.18632/oncotarget.5006.

68. Buj R, et al. Suppression of p16 Induces mTORC1-mediated nucleotide metabolic reprogramming. Cell Rep. 2019;28:1971-1980.e8.

69. Carreras CW. \& Santi DV. The catalytic mechanism and structure of thymidylate synthase. Ann Rev Biochem. 1995. https://doi.org/10.1146/annur ev.bi.64.070195.003445. 
70. Hazarika M, White RM, Johnson JR. \& Pazdur R. FDA drug approval summaries: pemetrexed (Alimta ${ }^{\circledR}$ ). Oncologist. 2004.https://doi.org/10.1634/ theoncologist.9-5-482.

71. Cohen MH, Justice R. \& Pazdur R. Approval summary: Pemetrexed in the initial treatment of advanced/metastatic non-small cell lung cancer. Oncologist. 2009.https://doi.org/10.1634/theoncologist.2009-0092.

72. Mairinger F. et al. Reduced folate carrier and folylpolyglutamate synthetase, but not thymidylate synthase predict survival in pemetrexedtreated patients suffering from malignant pleural mesothelioma. J Thorac Oncol. 2013.https://doi.org/10.1097/JTO.0b013e318287c224.

73. Ozasa $\mathrm{H}$. et al. Significance of thymidylate synthase for resistance to pemetrexed in lung cancer.Cancer Sci. 2010.https://doi.org/10.1111/j. 1349-7006.2009.01358.x.

74. Shan F, Liu YL, Wang Q. \& Shi YL. Thymidylate synthase predicts poor response to pemetrexed chemotherapy in patients with advanced breast cancer. Oncol Lett. 2018.https://doi.org/10.3892/ol.2018.8973.

75. Giovannetti, E. et al. Role of proton-coupled folate transporter in pemetrexed resistance of mesothelioma: Clinical evidence and new pharmacological tools. Ann Oncol. 2017.https://doi.org/10.1093/annonc/ mdx499.

76. Uemura T. et al. ABCC11/MRP8 confers pemetrexed resistance in lung cancer. Cancer Sci. 2010.https://doi.org/10.1111/j.1349-7006.2010. 01690.x.

77. Marangoni, E. et al. Capecitabine efficacy is correlated with tymp and rb1 expression in pdx established from triple-negative breast cancers. Clin. Cancer Res. 2018.https://doi.org/10.1158/1078-0432.CCR-17-3490.

78. Li W. \& Yue H. Thymidine phosphorylase: a potential new target for treat ing cardiovascular disease. Trends Cardiovasc Med. 2018.https://doi.org/ 10.1016/j.tcm.2017.10.003

79. Li Z, Zhang P, Ma Q, Wang D. \& Zhou T. Cisplatin-based chemoradiotherapy with 5-fluorouracil or pemetrexed in patients with locally advanced, unresectable esophageal squamous cell carcinoma: a retrospective analysis. Mol Clin Oncol. 2017.https://doi.org/10.3892/mco.2017.1222.

80. Chang PMH. et al. Transcriptome analysis and prognosis of ALDH isoforms in human cancer. Sci Rep. 2018.https://doi.org/10.1038/ s41598-018-21123-4.
81. Li, K. et al. ALDH2 repression promotes lung tumor progression via accumulated acetaldehyde and DNA damage. Neoplasia (United States). 2019.https://doi.org/10.1016/j.neo.2019.03.008.

82. Yang, M. et al. Methylation-induced silencing of ALDH2 facilitates lung adenocarcinoma bone metastasis by activating the MAPK pathway. Front Oncol. 2020.https://doi.org/10.3389/fonc.2020.01141.

83. Liu, W. et al. Fanconi anemia pathway as a prospective target for cancer intervention. Cell Biosci. 2020.https://doi.org/10.1186/ s13578-020-00401-7.

84. Yang Z, et al. 'Transcriptional silencing of ALDH2 confers a dependency on Fanconi anemia proteins in acute myeloid leukemia', Cancer Discovery. 2021. https://doi.org/10.1158/2159-8290.cd-20-1542.

85. Jacquemont C, Simon JA, D'Andrea AD. \& Taniguchi T. Non-specific chemical inhibition of the Fanconi anemia pathway sensitizes cancer cells to cisplatin. Mol Cancer. 2012.https://doi.org/10.1186/1476-4598-11-26.

86. Stecklein SR. \& Jensen RA. Identifying and exploiting defects in the fanconi anemia/BRCA pathway in oncology. Transl Res. 2012.https://doi.org/ 10.1016/j.trsl.2012.01.022.

87. Datta A. \& Brosh RM.New insights into DNA helicases as druggable targets for cancer therapy. Front Mol Biosci. 2018.https://doi.org/10.3389/ fmolb.2018.00059.

88. Serçin, Ö. et al. A solid-phase transfection platform for arrayed CRISPR screens. Mol Syst Biol. 2019.https://doi.org/10.15252/msb.20198983.

89. Drainas AP. et al. Genome-wide screens implicate loss of cullin ring ligase 3 in persistent proliferation and genome instability in TP53-deficient cells. Cell Rep.https://doi.org/10.1016/j.celrep.2020.03.029.

90. Guzmán C, Bagga M, Kaur A, Westermarck J. \& Abankwa D. ColonyArea: An ImageJ plugin to automatically quantify colony formation in clonogenic assays. PLoS One. 2014.https://doi.org/10.1371/journal.pone.00924 44.

\section{Publisher's Note}

Springer Nature remains neutral with regard to jurisdictional claims in published maps and institutional affiliations.
Ready to submit your research? Choose BMC and benefit from:

- fast, convenient online submission

- thorough peer review by experienced researchers in your field

- rapid publication on acceptance

- support for research data, including large and complex data types

- gold Open Access which fosters wider collaboration and increased citations

- maximum visibility for your research: over $100 \mathrm{M}$ website views per year

At BMC, research is always in progress.

Learn more biomedcentral.com/submissions 